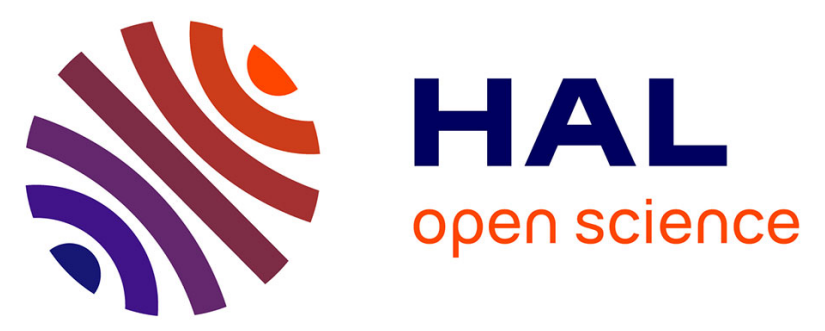

\title{
PEPPSI-Type Palladium-NHC Complexes: Synthesis, Characterization, and Catalytic Activity in the Direct C5-Arylation of 2-Substituted Thiophene Derivatives with Aryl Halides
}

M. Kaloğlu, I. Özdemir, V. Dorcet, Christian Bruneau, H. Doucet

\section{To cite this version:}

M. Kaloğlu, I. Özdemir, V. Dorcet, Christian Bruneau, H. Doucet. PEPPSI-Type Palladium-NHC Complexes: Synthesis, Characterization, and Catalytic Activity in the Direct C5-Arylation of 2Substituted Thiophene Derivatives with Aryl Halides. European Journal of Inorganic Chemistry, 2017, 2017 (10), pp.1382-1391. 10.1002/ejic.201601452 . hal-01515148

HAL Id: hal-01515148

https://hal-univ-rennes1.archives-ouvertes.fr/hal-01515148

Submitted on 6 Jul 2017

HAL is a multi-disciplinary open access archive for the deposit and dissemination of scientific research documents, whether they are published or not. The documents may come from teaching and research institutions in France or abroad, or from public or private research centers.
L'archive ouverte pluridisciplinaire HAL, est destinée au dépôt et à la diffusion de documents scientifiques de niveau recherche, publiés ou non, émanant des établissements d'enseignement et de recherche français ou étrangers, des laboratoires publics ou privés. 


\title{
PEPPSI-Type Palladium-NHC Complexes: Synthesis, Characterization, and Catalytic Activity in the Direct C5-Arylation of 2-Substituted Thiophene Derivatives with Aryl Halides
}

\author{
Murat Kaloğlu, ${ }^{[a, b]}$ İsmail Özdemir ${ }^{\star[a, b]}$ Vincent Dorcet, ${ }^{[c]}$ Christian Bruneau, ${ }^{[\mathrm{c}]}$ and Henri Doucet ${ }^{[\mathrm{c}]}$
}

\begin{abstract}
Benzimidazolium salts (2a-f) having their two nitrogen atoms substituted by different alkyl groups have been synthesized in high yields. The benzimidazolium salts were readily converted into the corresponding PEPPSI-type palladium-NHC complexes (3a-f) (PEPPSI = pyridine-enhanced precatalyst preparation, stabilisation, and initiation). The structures of all compounds were characterized by ${ }^{1} \mathrm{H}$ NMR, ${ }^{13} \mathrm{C}$ NMR, IR and elemental analysis techniques, which supported the proposed structures. The molecular structure of the complex $3 \mathbf{f}$ was determined by single-crystal X-ray diffraction. The catalytic activity of PEPPSI-type palladium-NHC complexes was evaluated in the direct C5-arylation of 2-substituted thiophene derivatives with various aryl halides. This arylation occured efficiently and selectively at the C5-position of 2-substituted thiophene derivatives.
\end{abstract}

\section{Introduction}

The use of $\mathrm{N}$-heterocyclic carbenes (NHCs) as ligands for transition metals was first described in 1968 by Öfele ${ }^{[1]}$ and Wanzlick. ${ }^{[2]}$ The development of metal-NHC complexes by Lappert $^{[3]}$ in the early 1970 s and the isolation of the first stable free NHC by Arduengo and co-workers ${ }^{[4]}$ in 1991 set the scene for an ever-growing interest and advancement in the field of NHC chemistry. Shortly thereafter, NHCs have been utilized extensively as ligands of transition metal complexes in organometallic chemistry and homogeneous catalysis. ${ }^{[5-17]}$

Since the initial work by Ohta and co-workers, ${ }^{[18]}$ transition metal-catalyzed direct arylation reactions have been rapidly developing for the synthesis of a wide range of heteroarenes and it still receives much interest from academic and industrial research groups. ${ }^{[19-28]}$

The direct arylation of heteroarenes with aryl halides has become the most valuable method for the formation of $\mathrm{C}\left(\mathrm{sp}^{2}\right)$ -

[a] Department of Chemistry, Faculty of Science and Art, Inönü University

44280 Malatya, Turkey

E-mail: ismail.ozdemir@inonu.edu.tr

https://www.inonu.edu.tr/

[b] Catalysis Research and Application Center, Inönü University. 44280 Malatya, Turkey

[c] UMR 6226 CNRS-Université de Rennes 1, Institut des Sciences Chimiques de Rennes, Campus de Beaulieu. 35042 Rennes, France

Supporting information for this article is given via a link at the end of the document.
$\mathrm{C}\left(\mathrm{sp}^{2}\right)$ bonds in contemporary organic synthesis because of the numerous applications of heteroaromatic compounds as biologically active compounds and functional materials. ${ }^{[2,30]}$ Thiophene derivatives show valuable biological activities and present considerable interest in pharmaceutical chemistry. As selected examples, Canagliflozin ${ }^{[31 a]}$ is a drug for treatment of type-2 diabetes, Evista ${ }^{[31 b]}$ is used for the prevention and treatment of osteoporosis, Saviprazole ${ }^{[31 c]}$ is a gastric proton pump inhibitor, Tiflucarbine ${ }^{[31 \mathrm{~d}]}$ displays antidepressant properties and Motapizone $e^{[31 e]}$ is used against platelet aggregation. Because of these properties, the discovery of simple and direct accesses to thiophene derivatives remains an important challenge for organic chemists.
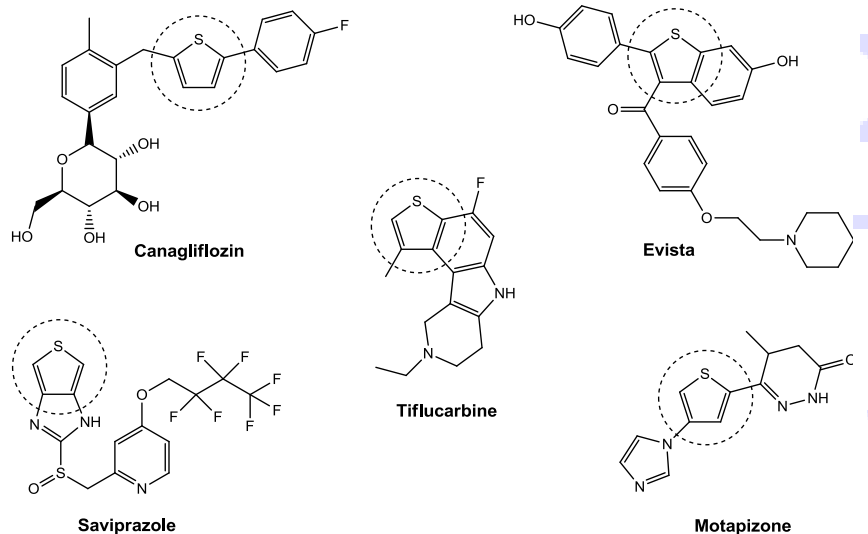

Figure 1. Examples of biologically active thiophene derivatives.

Palladium-catalyzed direct arylation of $\mathrm{C}-\mathrm{H}$ bonds of thiophenes with aryl halides is known to occur preferentially at the $\alpha$-positions to the sulfur atom (C2 and/or C5) following the typical reactivity profiles of the thiophene ring (Scheme 1). ${ }^{\text {[2] }}$

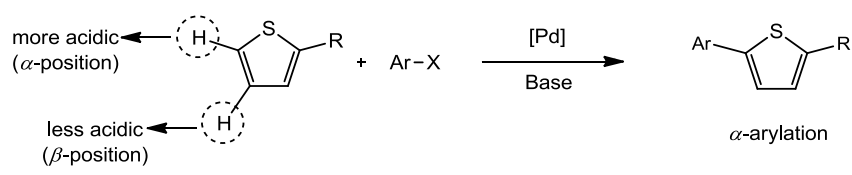

Scheme 1. Pd-catalyzed direct C5-arylation of 2-substituted thiophenes with aryl halides.

A number of palladium complexes with a single NHC ligand have proven to be useful catalysts in cross-coupling reactions of aryl halides. ${ }^{[33,34]}$ Among the most popular catalysts for such reactions are PEPPSI-type palladium-NHC complexes 
of the type $\left[\mathrm{PdX}_{2}(\mathrm{NHC})\right.$ (pyridine) $), \quad(\mathrm{X}=$ halide, PEPPSI= Pyridine-Enhanced Precatalyst Preparation, Stabilization and Initiation), which have gained real practical importance in numerous catalytic processes. ${ }^{[35]}$ In 2006, Organ et al. reported $^{[36]}$ easily-handled, air and moisture-stable $\mathrm{Pd}-\mathrm{NHC}$ complexes through the PEPPSI method that featured $\mathrm{Pd}(\mathrm{II})$ species bearing an NHC ligand, two halides, and a labile ligand such as 3-chloropyridine. A lot of work has been done by many groups since 2006 on this type of complexes, ${ }^{[37]}$ but only a few examples deal with the direct arylation of heteroarenes. ${ }^{[38]}$ In view of the above information and the growing interest of PEPPSI-type palladium-NHC complexes in catalysis we decided to investigate the catalytic activity of new members of this family in the direct C5-arylation of 2-substitued thiophenes.

We now describe the synthesis and characterization of the benzimidazolium salts (2a-f) as NHC species, and their corresponding PEPPSI-type palladium-NHC complexes (3a-f). These compounds were characterized by ${ }^{1} \mathrm{H}$ and ${ }^{13} \mathrm{C}$ NMR, IR and elemental analysis. The structure of the trans-dibromo[1-(3methoxypropyl)-3-(3,4,5-trimethoxybenzyl)benzimidazol-2-ylidene](pyridine)palladium(II) complex (3f) was determined by single-crystal $\mathrm{X}$-ray diffraction. We then examined the activity of the PEPPSI-type palladium-NHC complexes in the direct C5arylation of 2-substituted thiophene derivatives with various aryl bromides and aryl chlorides as coupling partners.

\section{Results and Discussion}

Preparation of benzimidazolium salts: The benzimidazolium salts (2a-f) were prepared by reacting $\mathrm{N}$-(3-methoxypropyl)benzimidazole (1) with various alkyl halides in DMF at $80^{\circ} \mathrm{C}$ for $36 \mathrm{~h}$ (Scheme 2). The benzimidazolium salts (2a-f) were air- and moisture-stable both in the solid state and in solution. The structures of the salts were determined by their characteristic spectroscopic data and elemental analyses. In the ${ }^{13} \mathrm{C}$ NMR spectra of 2a-f, the characteristic signals of the imino carbon, $(\mathrm{NCHN})$ were detected as typical singlets at 143.8, 143.8, 143.2, 143.8, 143.7 and 143.8 ppm, respectively. The ${ }^{1} \mathrm{H}$ NMR signals of the $\mathrm{C}(2)-H$ protons were observed as sharp singlets at chemical shifts of $11.82,11.49,10.86,11.83,11.55$ and 11.68 ppm, respectively for $\mathbf{2 a - f}$, and further supported the assigned structures. These NMR values were in line with those found for other benzimidazolium salts of the literature ${ }^{[39]}$ The formation of the benzimidazolium salts were also evidenced by their IR spectra, which showed (CN) bond absorption at 1560, 1557, 1556, 1557, 1558 and $1594 \mathrm{~cm}^{-1}$ for the respective $\mathrm{CN}$ bond vibration of $\mathbf{2 a - f .}$

\section{Preparation of the PEPPSI-type palladium-NHC complexes:} The general procedure for the preparation of PEPPSI-type palladium-NHC complexes (3a-f) according to the method reported by $\operatorname{Organ}^{[36]}$ is shown in Scheme 3 . Benzimidazolium salts (2a-f) were incorporated into the PEPPSI-type palladium$\mathrm{NHC}$ complexes (3a-f) by reaction with $\mathrm{PdCl}_{2}$ in refluxing pyridine in the presence of $\mathrm{K}_{2} \mathrm{CO}_{3}$ as a base and a large excess of $\mathrm{KBr}$ for $16 \mathrm{~h}$. These complexes, which were stable both in solution and in solid state against air, light and moisture, were soluble in different solvents such as dimethylsulfoxide, chloroform, dichloromethane and diethylether. The absence of the characteristic signals of the imino carbon (143-144 ppm) and the acidic imino proton (10-12 ppm) in ${ }^{13} \mathrm{C}$ NMR and ${ }^{1} \mathrm{H}$ NMR, which were present in the salts (2a-f) suggested the formation of the NHC-carbenes and their coordination to form the PEPPSItype palladium-NHC complexes. In addition, the characteristic carbenic carbons in compounds 3a-f appeared in the ${ }^{13} \mathrm{C}$ NMR spectra as deshielded singlets at 163.6, 162.2, 163.1, 163.1, 163.1 and $162.0 \mathrm{ppm}$, respectively. The IR data also clearly indicated the presence of $(\mathrm{CN})$ vibration at 1408, 1408, 1425, 1405,1412 and $1410 \mathrm{~cm}^{-1}$ for $3 a-f$. The formation of carbenes is correlated by a shift of the $(\mathrm{CN})$ vibration from $1556-1594 \mathrm{~cm}^{-1}$ in the benzimidazolium salts to $1408-1425 \mathrm{~cm}^{-1}$ in the coordinated carbenes.

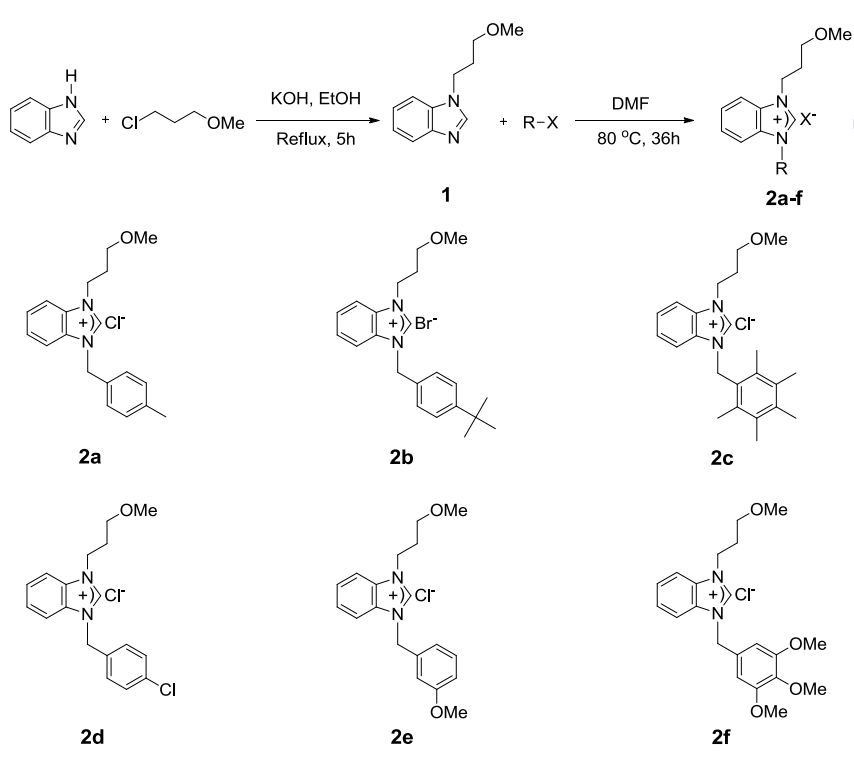

Scheme 2. Synthesis of the benzimidazolium salts (2a-f).

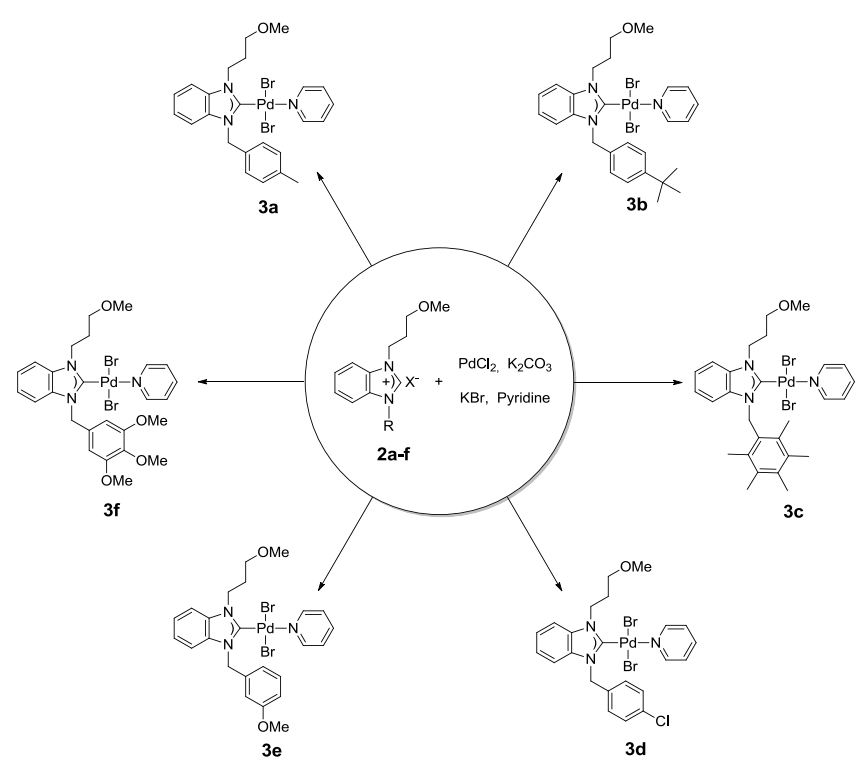

Scheme 3. Synthesis of the PEPPSI-type palladium-NHC complexes (3a-f). 
Structural characterization of complex 3f: Single crystals of complex $\mathbf{3 f}$ suitable for diffraction study were obtained by slow diffusion of $n$-pentane into a dichloromethane solution of complex 3f. The molecular structure of complex $\mathbf{3 f}$ has been confirmed by $\mathrm{X}$-ray single-crystal analyses. This complex crystallizes in a centrosymmetric monoclinic $P 12_{1} / c 1$ system, and adopts a square planar geometry. The carbene and the pyridine ligands are in trans-position with respective distances to the palladium centre of $1.963(7) \AA$ and $2.109(6) \AA$. The molecular structure of complex $\mathbf{3 f}$ is shown in Figure 1, and selected bond lengths and angles are summarized in Table 1.

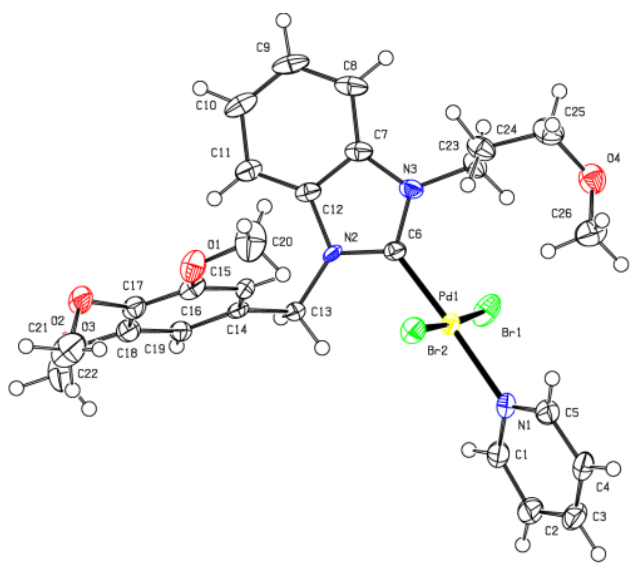

Figure 2. Perspective view of the molecular structure of $\mathbf{3 f}$.
Table 1. Selected bond lengths $[\AA]$ and angles $\left[^{\circ}\right]$ for complex $\mathbf{3 f}$.

\begin{tabular}{cccc}
\hline Pd1-C6 & $1.963(7)$ & N1-Pd1-Br1 & $92.03(16)$ \\
Pd1-N1 & $2.109(6)$ & N1-Pd1-Br2 & $91.21(16)$ \\
Pd1-Br1 & $2.4180(9)$ & Br1-Pd1-Br2 & $176.27(4)$ \\
Pd1-Br2 & $2.4265(9)$ & C5-N1-Pd1 & $123.0(5)$ \\
C6-Pd1-N1 & $177.0(3)$ & C1-N1-Pd1 & $118.9(5)$ \\
C6-Pd1-Br1 & $87.70(19)$ & N2-C6-Pd1 & $125.7(5)$ \\
C6-Pd1-Br2 & $89.17(19)$ & N3-C6-Pd1 & $127.4(5)$ \\
\hline
\end{tabular}

Direct C5-arylation of 2-substituted thiophene derivatives: In order to screen the experimental conditions, we selected the complex $\mathbf{3 f}$ as the catalyst. In all of complexes, methoxypropyl group linked to the nitrogen atom of the benzimidazole ring has an oxygen atom oriented toward the metal center. This orientation has also been proven for complex $\mathbf{3 f}$ by X-ray analysis (see Figure 2). For this reason, we think that this group could be hamilable effect throughout the catalytic cycle. We also selected the 2-acetylthiophene as model heteroaromatic substrate with a blocked C2-position (Scheme 4). The results of the reaction parameters including solvent, base, temperature and catalyst loading are gathered in Table 2 .

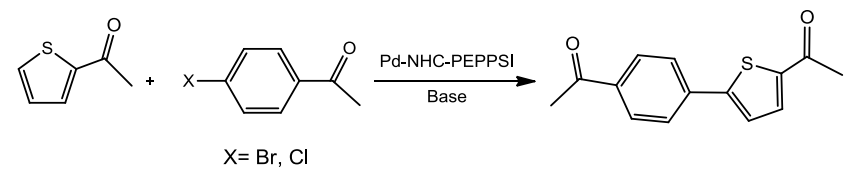

Scheme 4. Pd-NHC-PEPPSI-catalyzed direct C5-arylation of 2-acetylthiophene with 4-chloroacetophenone and 4-bromoacetophenone.

Table 2. Influence of the reaction conditions for Pd-NHC-PEPPSI catalyzed direct C5-arylation of 2-acetylthiophene with 4-chloro- and 4-bromoacetophenone. ${ }^{\text {[a] }}$

\begin{tabular}{|c|c|c|c|c|c|c|c|c|}
\hline Entry & $\begin{array}{c}\text { Pd-NHC-PEPPSI } \\
(\mathrm{mol} \%)\end{array}$ & $x$ & Solvent & Base & Time (h) & $\begin{array}{l}\text { Temp. } \\
\left({ }^{\circ} \mathrm{C}\right)\end{array}$ & $\begin{array}{c}\text { Conversion }{ }^{[\mathrm{b}]} \\
(\%)\end{array}$ & $\begin{array}{c}\text { Yield }^{[\mathrm{c}]} \\
(\%)\end{array}$ \\
\hline 1 & $3 f(1)$ & $\mathrm{Br}$ & DMAc & $\mathrm{NaOAc}$ & 2 & 120 & 61 & 54 \\
\hline 2 & $3 f(1)$ & $\mathrm{Br}$ & DMF & $\mathrm{NaOAc}$ & 2 & 120 & 34 & 30 \\
\hline 3 & $3 f(1)$ & $\mathrm{Br}$ & Toluene & $\mathrm{NaOAc}$ & 2 & 120 & 47 & 41 \\
\hline 4 & $3 f(1)$ & $\mathrm{Br}$ & DMAC & $\mathrm{K}_{2} \mathrm{CO}_{3}$ & 2 & 120 & 31 & 22 \\
\hline 5 & $3 f(1)$ & $\mathrm{Br}$ & DMF & $\mathrm{K}_{2} \mathrm{CO}_{3}$ & 2 & 120 & 19 & 11 \\
\hline 6 & $3 f(1)$ & $\mathrm{Br}$ & Toluene & $\mathrm{K}_{2} \mathrm{CO}_{3}$ & 2 & 120 & 24 & 18 \\
\hline 7 & $3 f(1)$ & $\mathrm{Br}$ & DMAc & KOAC & 2 & 120 & 86 & 71 \\
\hline 8 & $3 f(1)$ & $\mathrm{Br}$ & DMF & KOAC & 2 & 120 & 46 & 37 \\
\hline 9 & $3 f(1)$ & $\mathrm{Br}$ & Toluene & KOAC & 2 & 120 & 58 & 49 \\
\hline 10 & $3 f(1)$ & $\mathrm{Br}$ & DMAc & KOAC & 2 & 130 & 91 & 84 \\
\hline 11 & $3 f(1)$ & $\mathrm{Br}$ & DMAC & KOAC & 2 & 150 & 100 & 91 \\
\hline 12 & $3 f(0.5)$ & $\mathrm{Br}$ & DMAC & $\mathrm{KOAC}$ & 2 & 150 & 81 & 78 \\
\hline 13 & $3 f(1)$ & $\mathrm{Br}$ & DMAC & KOAC & 1 & 150 & 84 & 78 \\
\hline 14 & $3 f(1)$ & $\mathrm{Cl}$ & DMAC & KOAC & 2 & 150 & 14 & 8 \\
\hline 15 & $3 f(1)$ & $\mathrm{Cl}$ & DMAC & KOAC & 5 & 150 & 24 & 12 \\
\hline 16 & $3 f(1)$ & $\mathrm{Cl}$ & DMAC & KOAC & 10 & 150 & 43 & 34 \\
\hline 17 & $3 f(1)$ & $\mathrm{Cl}$ & DMAC & KOAC & 15 & 150 & 68 & 58 \\
\hline 18 & $3 f(1)$ & $\mathrm{Cl}$ & DMAC & KOAC & 20 & 150 & 87 & 79 \\
\hline 19 & $3 f(1)$ & $\mathrm{Cl}$ & DMAC & KOAC & 20 & 120 & 64 & 36 \\
\hline 20 & $3 f(1)$ & $\mathrm{Cl}$ & DMAC & KOAC & 20 & 130 & 71 & 51 \\
\hline 21 & $3 f(1)$ & $\mathrm{Cl}$ & DMAc & KOAC & 25 & 150 & 90 & 81 \\
\hline
\end{tabular}

[a] Conditions: 2-acetylthiophene $(2 \mathrm{mmol})$, aryl halide $(1 \mathrm{mmol})$, base $(2 \mathrm{mmol})$, solvent $(2 \mathrm{~mL})$. [b] Conversions were calculated according to aryl halide by GC and GC-MS. [c] Isolated yields. 
When DMF or toluene were used as solvent, the reaction gave low conversion of only $19-58 \%$ with $\mathrm{NaOAc}, \mathrm{K}_{2} \mathrm{CO}_{3}$ or $\mathrm{KOAc}$ as base after $2 \mathrm{~h}$ at $120^{\circ} \mathrm{C}$ (Table 2, entries 2, 3, 5, 6, 8, 9). DMAc proved to be the best tested solvent after $2 \mathrm{~h}$ at $120^{\circ} \mathrm{C}$. In this solvent decreasing the reaction temperature from $150{ }^{\circ} \mathrm{C}$ to $120{ }^{\circ} \mathrm{C}$ had a detrimental effect on the conversion (Table 2, entries $1,4,7,10,11)$. In the presence of $0.5 \mathrm{~mol} \%$ of $\mathbf{3} f$ as the catalyst, KOAc as the base, DMAc as the solvent and 4-bromoacetophenone as the coupling partner at $150{ }^{\circ} \mathrm{C}$ for $2 \mathrm{~h}$, the C5-arylated product was obtained in $78 \%$ isolated yield (Table 2, entry 12). When the reaction time was reduced to $1 \mathrm{~h}$, the reaction gave low conversion of only $84 \%$ (Table 2, entry 13 ). Finally, the best conditions leading to full conversion of 4-bromoacetophenone with high selectivity in favor of the C5arylated product were obtained when the reaction was carried out in DMAc in the presence of 2 equiv. of KOAc at $150{ }^{\circ} \mathrm{C}$ for 2 h (Table 2, entry 11).

When the less reactive 4-chloroacetophenone was used as substrate in the presence of $1 \mathrm{~mol} \%$ of catalyst $\mathbf{3 f}$ and KOAc as the base at $150{ }^{\circ} \mathrm{C}$, the conversions increased depending on the reaction time (Table 2 , entries $14,15,16,17$ ). Interestingly, up to $87 \%$ conversion with $79 \%$ isolated yield were obtained, but for this the reaction required a longer reation time of $20 \mathrm{~h}$ (Table 2, entry 18). The reaction temperature decreasing from
$150{ }^{\circ} \mathrm{C}$ to $120{ }^{\circ} \mathrm{C}$ had a detrimental effect on the conversion (Table 2, entries 19 and 20). When the reaction time was increased from $20 \mathrm{~h}$ to $25 \mathrm{~h}$ at $150{ }^{\circ} \mathrm{C}$, the very close conversion of 4-chloroacetophenone was obtained (Table 2, entry 21).

Finally, the scope of the direct C5-arylation of two 2-substituted thiophene substrates was investigated with various aryl halides, including five (hetero)aryl bromides (Tables 3 and 5) and four aryl chlorides (Tables 4 and 6) applying our best experimental conditions. Only a minor effect of the carbene ligand on the Pd-PEPPSI complex was observed for the coupling of aryl bromides with thiophene derivatives. In all cases, except in a few cases with complex $\mathbf{3 f}$, high conversions of the aryl bromide were observed, and the coupling products were isolated in high yields. At elevated temperature, the oxidative addition of the aryl bromide to palladium is generally easy and does not require the use of very specific ligands. A more important effect of the nature of the ligand was expected for the coupling of aryl chlorides with the thiophenes derivatives (Tables 4 and 6). ${ }^{[32]}$ Indeed, again in all cases the coupling products were produced in good yields. Surprisingly, similar yields were obtained for the coupling of electron-deficient aryl chlorides (Tables 4 and 6, entries 13-24) as with chlorobenzene (Table 4 and 6 , entries 1-6). In this case, the presence of the NHCligands on palladium likely favors the oxidative addition step.

Table 3. Pd-NHC-PEPPSI-catalyzed direct C5-arylation of 2-acetylthiophene with aryl bromides. ${ }^{[\mathrm{a}]}$

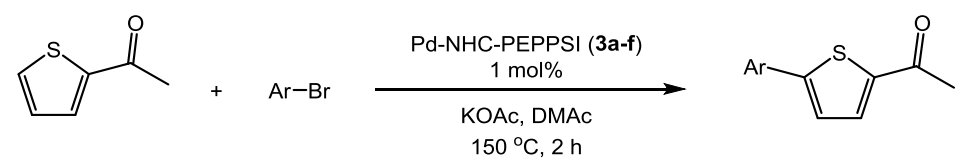

\begin{tabular}{|c|c|c|c|c|c|}
\hline Entry & Aryl bromide & Catalyst & Product & Conversion $^{[0]}(\%)$ & Yield $^{[[]}(\%)$ \\
\hline 1 & & $3 a$ & & 79 & 71 \\
\hline 2 & & $3 b$ & & 83 & 75 \\
\hline 3 & & 3c & & 90 & 88 \\
\hline 4 & & $3 d$ & & 96 & 85 \\
\hline 5 & & $3 e$ & & 85 & 80 \\
\hline 6 & & $3 f$ & & 90 & 87 \\
\hline 7 & & $3 a$ & & 91 & 82 \\
\hline 8 & & $3 b$ & & 83 & 79 \\
\hline 9 & & $3 c$ & & 100 & 92 \\
\hline 10 & & 3d & & 97 & 90 \\
\hline 11 & & $3 e$ & & 96 & 84 \\
\hline 12 & & $3 f$ & & 100 & 90 \\
\hline 13 & & $3 a$ & & 76 & 70 \\
\hline 14 & & $3 b$ & & 98 & 88 \\
\hline 15 & & $3 c$ & & 93 & 87 \\
\hline 16 & & $3 d$ & & 87 & 84 \\
\hline 17 & & $3 e$ & & 86 & 81 \\
\hline 18 & & $3 f$ & & 100 & 91 \\
\hline $\begin{array}{l}19 \\
20\end{array}$ & & $\begin{array}{l}3 a \\
3 \mathbf{b}\end{array}$ & & $\begin{array}{l}86 \\
83\end{array}$ & $\begin{array}{l}82 \\
74\end{array}$ \\
\hline 21 & & $3 c$ & & 100 & 93 \\
\hline 22 & & 3d & & 94 & 91 \\
\hline 23 & $\mathrm{NC}$ & $3 e$ & & 91 & 83 \\
\hline 24 & & $3 f$ & & 100 & 85 \\
\hline 25 & & $3 a$ & & 91 & 84 \\
\hline 26 & & $\begin{array}{l}3 a \\
3 b\end{array}$ & & 87 & $\begin{array}{l}04 \\
81\end{array}$ \\
\hline 27 & & $3 c$ & & 98 & 87 \\
\hline 28 & & $3 d$ & & 100 & 92 \\
\hline 29 & & $3 e$ & & 93 & 88 \\
\hline 30 & & $3 f$ & & 98 & 87 \\
\hline
\end{tabular}

[a] Conditions: Pd-NHC-PEPPSI $(0.01 \mathrm{mmol}), 2$-acetylthiophene $(2 \mathrm{mmol})$, aryl bromide $(1 \mathrm{mmol}), \mathrm{KOAc}(2 \mathrm{mmol}), \mathrm{DMAc}(2 \mathrm{~mL}), 150{ }^{\circ} \mathrm{C}, 2 \mathrm{~h}$

[b] Conversions were calculated according to aryl bromide by GC. [c] Isolated yields. 


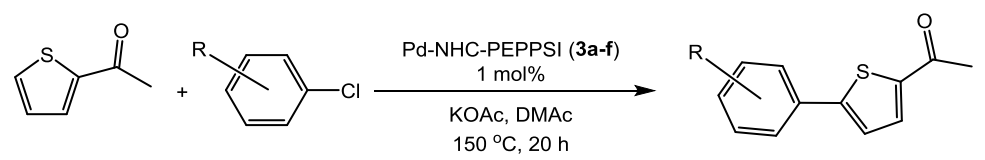

\begin{tabular}{|c|c|c|c|c|c|}
\hline Entry & Aryl chloride & Catalyst & Product & Conversion $^{[b]}(\%)$ & Yield $^{[c]}(\%)$ \\
\hline 1 & & $3 a$ & & 87 & 78 \\
\hline 2 & & $3 b$ & & 59 & 54 \\
\hline 3 & & $3 c$ & & 51 & 44 \\
\hline 4 & & $3 d$ & & 63 & 57 \\
\hline 5 & & $3 e$ & & 74 & 71 \\
\hline 6 & & $3 f$ & & 83 & 76 \\
\hline 7 & & $3 a$ & & 71 & 64 \\
\hline 8 & & $3 b$ & & 63 & 57 \\
\hline 9 & & $3 c$ & & 59 & 51 \\
\hline 10 & & $3 d$ & & 68 & 66 \\
\hline 11 & & $3 e$ & & 78 & 69 \\
\hline 12 & & $3 f$ & & 79 & 61 \\
\hline 13 & & $3 a$ & & 78 & 76 \\
\hline 14 & & $3 b$ & & 86 & 79 \\
\hline 15 & & 3c & & 50 & 41 \\
\hline 16 & & $3 d$ & & 76 & 69 \\
\hline 17 & & $3 e$ & & 64 & 60 \\
\hline 18 & & $3 f$ & & 87 & 79 \\
\hline 19 & & $3 a$ & & 80 & 74 \\
\hline 20 & & $3 b$ & & 94 & 88 \\
\hline 21 & & $3 c$ & & 66 & 61 \\
\hline 22 & 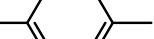 & 3d & & 88 & 80 \\
\hline 23 & & $3 e$ & & 73 & 64 \\
\hline 24 & & $3 f$ & & 81 & 72 \\
\hline
\end{tabular}

[a] Conditions: Pd-NHC-PEPPSI $(0.01 \mathrm{mmol}), 2$-acetylthiophene $(2 \mathrm{mmol})$, aryl chloride $(1 \mathrm{mmol}), \mathrm{KOAc}(2 \mathrm{mmol}), \mathrm{DMAc}(2 \mathrm{~mL}), 150{ }^{\circ} \mathrm{C}, 20 \mathrm{~h}$. [b] Conversions were calculated according to aryl chloride by GC. [c] Isolated yields.

Table 5. Pd-NHC-PEPPSI catalyzed direct C5-arylation of 2-cyanomethylthiophene by using aryl bromides. ${ }^{\text {[a] }}$

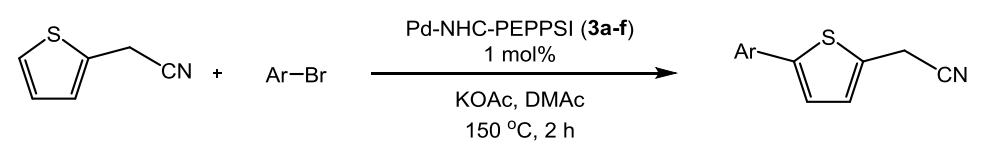

\begin{tabular}{|c|c|c|c|c|c|}
\hline Entry & Aryl bromide & Catalyst & Product & Conversion $^{[0]}(\%)$ & Yield $^{[C]}(\%)$ \\
\hline 1 & & $3 a$ & & 88 & 84 \\
\hline 2 & & $3 b$ & & 78 & 71 \\
\hline 3 & & $3 c$ & & 85 & 79 \\
\hline 4 & & 3d & & 74 & 69 \\
\hline 5 & & $3 e$ & & 89 & 85 \\
\hline 6 & & $3 f$ & & 79 & 72 \\
\hline 7 & & $3 a$ & & 100 & 91 \\
\hline 8 & & $3 b$ & & 90 & 86 \\
\hline 9 & & $3 c$ & & 100 & 88 \\
\hline 10 & & 3d & & 86 & 80 \\
\hline 11 & & $3 e$ & & 90 & 71 \\
\hline 12 & & $3 f$ & & 84 & 78 \\
\hline 13 & & $3 a$ & & 89 & 81 \\
\hline 14 & & $3 b$ & & 88 & 77 \\
\hline 15 & & $3 c$ & & 84 & 77 \\
\hline 16 & & 3d & & 93 & 87 \\
\hline 17 & & $3 e$ & & 97 & 89 \\
\hline 18 & & $3 f$ & & 73 & 68 \\
\hline 19 & & $3 a$ & & 94 & 89 \\
\hline 20 & & $3 b$ & & 76 & 71 \\
\hline 21 & & $3 c$ & & 83 & 78 \\
\hline 22 & & 3d & & 84 & 79 \\
\hline 23 & $\mathrm{~N}$ & $3 e$ & & 81 & 78 \\
\hline 24 & & $3 f$ & & 61 & 56 \\
\hline 25 & & $3 a$ & & 93 & 86 \\
\hline 26 & & $3 b$ & & 87 & 84 \\
\hline 27 & & $3 c$ & & 77 & 74 \\
\hline 28 & & $3 d$ & & 88 & 82 \\
\hline 29 & & $3 e$ & & 84 & 77 \\
\hline 30 & & $3 f$ & & 75 & 73 \\
\hline
\end{tabular}

[a] Conditions: Pd-NHC-PEPPSI (0.01 mmol), 2-cyanomethylthiophene $(2 \mathrm{mmol})$, aryl bromide $(1 \mathrm{mmol}), \mathrm{KOAc}(2 \mathrm{mmol}), \mathrm{DMAc}(2 \mathrm{~mL}), 150{ }^{\circ} \mathrm{C}, 2 \mathrm{~h}$. [b] Conversions were calculated according to aryl bromide by GC. [c] Isolated yields. 


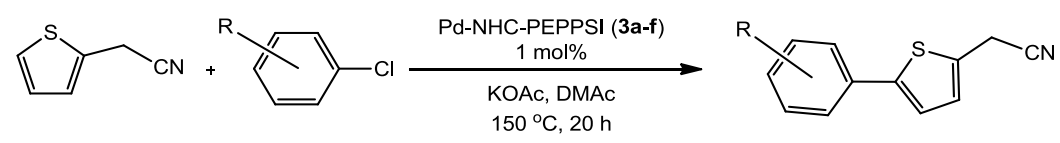

\begin{tabular}{|c|c|c|c|c|c|}
\hline Entry & Aryl chloride & Catalyst & Product & Conversion $^{[\mathrm{b}]}(\%)$ & Yield $^{[\mathrm{CI}}(\%)$ \\
\hline 1 & & $3 a$ & & 73 & 64 \\
\hline 2 & & $3 b$ & & 71 & 65 \\
\hline 3 & & $3 c$ & & 78 & 74 \\
\hline 4 & & 3d & & 71 & 68 \\
\hline 5 & & $3 e$ & & 83 & 77 \\
\hline 6 & & $3 f$ & & 70 & 68 \\
\hline 7 & & $3 a$ & & 62 & 58 \\
\hline 8 & & $3 b$ & & 57 & 54 \\
\hline 9 & & $3 c$ & & 49 & 44 \\
\hline 10 & & 3d & & 67 & 64 \\
\hline 11 & & $3 e$ & & 71 & 63 \\
\hline 12 & & $3 f$ & & 64 & 59 \\
\hline 13 & & $3 \mathbf{a}$ & & 85 & 79 \\
\hline 14 & & $3 b$ & & 78 & 76 \\
\hline 15 & & $3 c$ & & 74 & 70 \\
\hline 16 & & $3 d$ & & 88 & 84 \\
\hline 17 & & $3 e$ & & 74 & 60 \\
\hline 18 & & $3 f$ & & 70 & 61 \\
\hline 19 & & $3 a$ & & 69 & 65 \\
\hline 20 & & $3 b$ & & 63 & 58 \\
\hline 21 & & $3 c$ & & 76 & 72 \\
\hline 22 & $\lambda$ & 3d & & 75 & 70 \\
\hline 23 & & $3 e$ & & 75 & 73 \\
\hline 24 & & $3 f$ & & 78 & 74 \\
\hline
\end{tabular}

[a] Conditions: Pd-NHC-PEPPSI (0.01 mmol), 2-cyanomethylthiophene (2 mmol), aryl chloride (1 mmol), $\mathrm{KOAc}(2 \mathrm{mmol}), \mathrm{DMAc}(2 \mathrm{~mL}), 150{ }^{\circ} \mathrm{C}, 20 \mathrm{~h}$.

[b] Conversions were calculated according to aryl chloride by GC. [c] Isolated yields.

\section{Conclusions}

In summary, we have prepared a series of new benzimidazolium salts from $\mathrm{N}$-substituted benzimidazole. These benzimidazolium salts were metallated with $\mathrm{PdCl}_{2}$ in pyridine to give easilyhandled, air and moisture stable new PEPPSI-type palladium$\mathrm{NHC}$ complexes. Then, all benzimidazolium salts and PEPPSItype palladium-NHC complexes were characterized using different spectroscopic and analytical techniques. The structure of complex $3 \mathbf{f}$ was determined by single-crystal X-ray diffraction. The PEPPSI-type palladium-NHC complexes were used in the direct C5-arylation of 2-substituted thiophenes.

The catalytic systems generated from these PEPPSI-type palladium-NHC complexes in the presence of KOAc as the base and DMAc as the solvent at $150{ }^{\circ} \mathrm{C}$ were very efficient at $1 \mathrm{~mol} \%$ catalyst loading for the selective $\mathrm{C}-\mathrm{C}$ bond formation from aryl halides. When the catalytic studies were evaluated, it was found that all of the complexes were suitable for the direct C5-arylation of 2-substituted thiophene derivatives with aryl bromides. With these catalysts, even non-activated aryl chlorides such as chlorobenzene or 4-chlorotoluene were coupled with thiophenes derivatives in good yields.

It is clear that, the use of PEPPSI-type palladium-NHC complexes as catalysts in the direct arylation of thiophenes was very limited in the literature. Also, this study has some advantages such as low catalyst loading and less reaction time when compered with the previously reported studies on the arylation of thiophene derivatives. ${ }^{[29 f, 30 j, 32 \mathrm{c}, 38 \mathrm{c}]}$

\section{Experimental Section}

General Methods: All manipulations were performed in Schlenk type flasks under argon. All chemical reactants were obtained from commercial sources. The solvents used were purified by distillation over the drying agents indicated and were transferred under argon. Pd-NHCPEPPSI complexes were prepared according to known methods in the literature. ${ }^{[37]}$ DMAc analytical grade $(99 \%)$ was not distilled before use. KOAc (99\%) was employed. ${ }^{1} \mathrm{H}$ NMR (used 300 and $500 \mathrm{MHz}$ ) and ${ }^{13} \mathrm{C}$ NMR (used 75 and $125 \mathrm{MHz}$ ) spectra were recorded in $\mathrm{CDCl}_{3}$. Chemical shifts $(\delta)$ are reported in ppm relative to $\mathrm{CDCl}_{3} .{ }^{1} \mathrm{H}$ NMR spectra are referenced to residual protiated solvents $\left(\delta=7.26 \mathrm{ppm}\right.$ for $\left.\mathrm{CDCl}_{3}\right),{ }^{13} \mathrm{C}$ chemical shifts are reported relative to deuteriated solvents $(\delta=77.16$ $\mathrm{ppm}$ for $\mathrm{CDCl}_{3}$ ). IR spectra were recorded on ATR unit in the range of $400-4000 \mathrm{~cm}^{-1}$ with Perkin Elmer Spectrum 100 Spectrofotometer. Melting points were determined in glass capillaries under air with an Electrothermal-9200 melting point apparatus. Elemental analyses were performed by İnönü University Scientific and Technological Research Center (IBTAM). All catalytic reactions were monitored on an Agilent 6890 N GC and Schimadzu 2010 Plus GC-MS system by GC-FID with an HP- 5 column of $30 \mathrm{~m}$ length, $0.32 \mathrm{~mm}$ diameter, and $0.25 \mu \mathrm{m}$ film thickness.

General procedure for the preparation of benzimidazolium salts: For the preparation of $\mathrm{N}$-(3-methoxypropyl)benzimidazole (1), benzimidazole $(1.0 \mathrm{mmol})$ and potassium hydroxide $(1.0 \mathrm{mmol})$ were dissolved in ethyl alcohol $(50 \mathrm{~mL})$. 3-Methoxypropyl chloride $(1.0 \mathrm{mmol})$ was slowly added and the reaction mixture was stirred at room temperature for $1 \mathrm{~h}$. The solution was refluxed for $5 \mathrm{~h}$, then cooled to room temperature and the precipitated potassium chloride was removed by filtration. The solvent was removed by distillation. The crude product was then distilled under vacuum. For the preparation of the benzimidazolium salts (2a-f), $\mathrm{N}$-(3-methoxypropyl)benzimidazole (1), (1.0 mmol) was dissolved in dried dimethylformamide $(3 \mathrm{~mL})$ and the alkyl halide $(1.0 \mathrm{mmol})$ was added 
slowly. The reaction mixture was stirred at $80{ }^{\circ} \mathrm{C}$ for $36 \mathrm{~h}$ under argon. After completion of the reaction, all dimethylformamide was removed by vacuum and diethylether $(15 \mathrm{~mL})$ was added to obtain a white crystalline solid, which was filtered off. The solid was washed with diethylether $(3 \times$ $10 \mathrm{~mL}$ ) and dried under vacuum. The crude product was recrystallized from an ethanol/diethylether mixture $(1: 2, v / v)$.

1-(3-Methoxypropyl)-3-(4-methylbenzyl)benzimidazolium chloride (2a): Yield: $1.76 \mathrm{~g}, 91 \%$; mp: $130-131{ }^{\circ} \mathrm{C}$; ${ }^{1} \mathrm{H}$ NMR $\left(500 \mathrm{MHz}, \mathrm{CDCl}_{3}, 25\right.$ $\left.{ }^{\circ} \mathrm{C}\right): \delta 2.30\left(\mathrm{~s}, 3 \mathrm{H}, \quad \mathrm{NCH}_{2} \mathrm{C}_{6} \mathrm{H}_{4}\left(\mathrm{CH}_{3}\right)-4\right) ; 2.36(\mathrm{p}, J=5.6 \mathrm{~Hz}, 2 \mathrm{H}$, $\mathrm{NCH}_{2} \mathrm{CH}_{2} \mathrm{CH}_{2} \mathrm{OCH}_{3}$ ); 3.27 (s, 3H, $\mathrm{NCH}_{2} \mathrm{CH}_{2} \mathrm{CH}_{2} \mathrm{OCH}_{3}$ ); 3.49 (t, J=5.4 Hz, $2 \mathrm{H}, \mathrm{NCH}_{2} \mathrm{CH}_{2} \mathrm{CH}_{2} \mathrm{OCH}_{3}$ ); 4.74 (t, J=7.0 Hz, $2 \mathrm{H}, \mathrm{NCH}_{2} \mathrm{CH}_{2} \mathrm{CH}_{2} \mathrm{OCH}_{3}$ ); $5.84 \quad\left(\mathrm{~s}, \quad 2 \mathrm{H}, \quad \mathrm{NCH}_{2} \mathrm{C}_{6} \mathrm{H}_{4}\left(\mathrm{CH}_{3}\right)-4\right) ; \quad 7.15-7.76 \quad\left(\mathrm{~m}, \quad 8 \mathrm{H}, \quad \mathrm{NC}_{6} \mathrm{H}_{4} \mathrm{~N}\right.$, $\left.\mathrm{NCH}_{2} \mathrm{C}_{6} \mathrm{H}_{4}\left(\mathrm{CH}_{3}\right)-4\right) ; 11.82(\mathrm{~s}, 1 \mathrm{H}, \mathrm{NCHN}) .{ }^{13} \mathrm{C} \mathrm{NMR}\left(125 \mathrm{MHz}, \mathrm{CDCl}_{3}, 25\right.$ $\left.{ }^{\circ} \mathrm{C}\right): \delta 21.2 \quad\left(\mathrm{NCH}_{2} \mathrm{C}_{6} \mathrm{H}_{4}\left(\mathrm{CH}_{3}\right)-4\right) ; 29.7 \quad\left(\mathrm{NCH}_{2} \mathrm{CH}_{2} \mathrm{CH}_{2} \mathrm{OCH}_{3}\right) ; 45.0$ $\left(\mathrm{NCH}_{2} \mathrm{CH}_{2} \mathrm{CH}_{2} \mathrm{OCH}_{3}\right) ; \quad 51.3 \quad\left(\mathrm{NCH}_{2} \mathrm{C}_{6} \mathrm{H}_{4}\left(\mathrm{CH}_{3}\right)-4\right) ; \quad 58.8$ $\left(\mathrm{NCH}_{2} \mathrm{CH}_{2} \mathrm{CH}_{2} \mathrm{OCH}_{3}\right) ; 68.9\left(\mathrm{NCH}_{2} \mathrm{CH}_{2} \mathrm{CH}_{2} \mathrm{OCH}_{3}\right) ; 113.0$, 113.7, 126.9, $128.3, \quad 129.9, \quad 130.0, \quad 130.1, \quad 131.0, \quad 131.8, \quad 139.1 \quad\left(\mathrm{NC}_{6} \mathrm{H}_{4} \mathrm{~N}\right.$, $\left.\mathrm{NCH}_{2} \mathrm{C}_{6} \mathrm{H}_{4}\left(\mathrm{CH}_{3}\right)-4\right) ; 143.8(\mathrm{NCHN})$. IR $\left(\mathrm{cm}^{-1}\right) \mathrm{V}_{(\mathrm{CN})}$ : 1560; Anal. Calcd. for $\mathrm{C}_{19} \mathrm{H}_{23} \mathrm{CIN}_{2} \mathrm{O}: \mathrm{C}, 68.97 ; \mathrm{H}, 7.01 ; \mathrm{N}, 8.47$. Found: $\mathrm{C}, 68.78 ; \mathrm{H}, 7.02 ; \mathrm{N}$, 8.49 .

1-(3-Methoxypropyl)-3-(4-tert-butylbenzyl)benzimidazolium bromide (2b): Yield: $2.12 \mathrm{~g}, 87 \%$; mp: $161-162{ }^{\circ} \mathrm{C} ;{ }^{1} \mathrm{H} \mathrm{NMR}\left(300 \mathrm{MHz}, \mathrm{CDCl}_{3}, 25\right.$ $\left.{ }^{\circ} \mathrm{C}\right): \delta 1.19\left(\mathrm{~s}, 9 \mathrm{H}, \mathrm{NCH}_{2} \mathrm{C}_{6} \mathrm{H}_{4}\left(\mathrm{C}_{(}\left(\mathrm{CH}_{3}\right)_{3}\right)-4\right) ; 2.29(\mathrm{p}, J=5.6 \mathrm{~Hz}, 2 \mathrm{H}$, $\mathrm{NCH}_{2} \mathrm{CH}_{2} \mathrm{CH}_{2} \mathrm{OCH}_{3}$ ); 3.18 (s, 3H, $\mathrm{NCH}_{2} \mathrm{CH}_{2} \mathrm{CH}_{2} \mathrm{OCH}_{3}$ ); 3.42 (t, J=5.4 Hz, $2 \mathrm{H}, \mathrm{NCH}_{2} \mathrm{CH}_{2} \mathrm{CH}_{2} \mathrm{OCH}_{3}$ ); 4.67 (t, J= $7.0 \mathrm{~Hz}, 2 \mathrm{H}, \mathrm{NCH}_{2} \mathrm{CH}_{2} \mathrm{CH}_{2} \mathrm{OCH}_{3}$ ); $5.76 \quad\left(\mathrm{~s}, \quad 2 \mathrm{H}, \quad \mathrm{NCH}_{2} \mathrm{C}_{6} \mathrm{H}_{4}\left(\mathrm{C}_{(}\left(\mathrm{CH}_{3}\right)_{3}\right)-4\right) ; \quad 7.32-7.70 \quad\left(\mathrm{~m}, \quad 8 \mathrm{H}, \quad \mathrm{NC}_{6} \mathrm{H}_{4} \mathrm{~N}\right.$

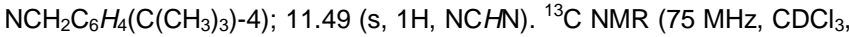

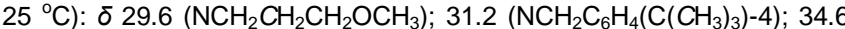
$\left(\mathrm{NCH}_{2} \mathrm{C}_{6} \mathrm{H}_{4}\left(\mathrm{C}\left(\mathrm{CH}_{3}\right)_{3}\right)-4\right) ; \quad 45.0 \quad\left(\mathrm{NCH}_{2} \mathrm{CH}_{2} \mathrm{CH}_{2} \mathrm{OCH}_{3}\right) ; \quad 51.0$ $\left(\mathrm{NCH}_{2} \mathrm{C}_{6} \mathrm{H}_{4}\left(\mathrm{C}\left(\mathrm{CH}_{3}\right)_{3}\right)-4\right) ; \quad 58.7 \quad\left(\mathrm{NCH}_{2} \mathrm{CH}_{2} \mathrm{CH}_{2} \mathrm{OCH}_{3}\right) ; \quad 68.9$ $\left(\mathrm{NCH}_{2} \mathrm{CH}_{2} \mathrm{CH}_{2} \mathrm{OCH}_{3}\right) ; 113.0,113.7,126.2,126.3,127.0,127.1,128.1$, $129.8,131.1,131.7,152.4\left(\mathrm{NC}_{6} \mathrm{H}_{4} \mathrm{~N}, \mathrm{NCH}_{2} \mathrm{C}_{6} \mathrm{H}_{4}\left(\mathrm{CH}_{3}\right)-4\right) ; 143.8(\mathrm{NCHN})$. IR $\left(\mathrm{cm}^{-1}\right) \mathrm{v}_{(\mathrm{CN})}$ : 1557 ; Anal. Calcd. for $\mathrm{C}_{22} \mathrm{H}_{29} \mathrm{BrN} \mathrm{Br}_{2} \mathrm{O}: \mathrm{C}, 63.31 ; \mathrm{H}, 7.00 ; \mathrm{N}$, 6.71. Found: C, 63.38; H, 7.12; N, 6.79.

1-(3-Methoxypropyl)-3-(2,3,4,5,6-pentamethylbenzyl)benzimidazolium chloride (2c): Yield: $1.55 \mathrm{~g}, 84 \%$; mp: $143-144{ }^{\circ} \mathrm{C} ;{ }^{1} \mathrm{H}$ NMR $(500$ $\left.\mathrm{MHz}, \mathrm{CDCl}_{3}, 25^{\circ} \mathrm{C}\right): \delta 2.20\left(\mathrm{p}, \mathrm{J}=5.3 \mathrm{~Hz}, 2 \mathrm{H}, \mathrm{NCH}_{2} \mathrm{CH}_{2} \mathrm{CH}_{2} \mathrm{OCH}_{3}\right) ; 2.23$, 2.26 and 2.28 (s, $\left.15 \mathrm{H}, \quad \mathrm{NCH}_{2} \mathrm{C}_{6}\left(\mathrm{CH}_{3}\right)_{5}-2,3,4,5,6\right) ; 3.20$ (s, $3 \mathrm{H}$, $\mathrm{NCH}_{2} \mathrm{CH}_{2} \mathrm{CH}_{2} \mathrm{OCH}$ ); 3.44 (t, J=5.5 Hz, $2 \mathrm{H}, \mathrm{NCH}_{2} \mathrm{CH}_{2} \mathrm{CH}_{2} \mathrm{OCH}_{3}$ ); 4.82 (t, $\left.J=6.9 \mathrm{~Hz}, 2 \mathrm{H}, \quad \mathrm{NCH}_{2} \mathrm{CH}_{2} \mathrm{CH}_{2} \mathrm{OCH}_{3}\right) ; 5.84\left(\mathrm{~s}, 2 \mathrm{H}, \quad \mathrm{NCH}_{2} \mathrm{C}_{6}\left(\mathrm{CH}_{3}\right)_{5}-\right.$ 2,3,4,5,6); 7.33-7.79 (m, 4H, NC $\left.6 H_{4} \mathrm{~N}\right) ; 10.86(\mathrm{~s}, 1 \mathrm{H}, \mathrm{NCHN}) .{ }^{13} \mathrm{C} \mathrm{NMR}$ $\left(125 \mathrm{MHz}, \mathrm{CDCl}_{3}, 25{ }^{\circ} \mathrm{C}\right): \delta 17.0,17.1$ and $17.3\left(\mathrm{NCH}_{2} \mathrm{C}_{6}\left(\mathrm{CH}_{3}\right)_{5-}\right.$ 2,3,4,5,6); $29.7\left(\mathrm{NCH}_{2} \mathrm{CH}_{2} \mathrm{CH}_{2} \mathrm{OCH}_{3}\right) ; 45.1\left(\mathrm{NCH}_{2} \mathrm{CH}_{2} \mathrm{CH}_{2} \mathrm{OCH}_{3}\right) ; 48.1$ $\left(\mathrm{NCH}_{2} \mathrm{C}_{6}\left(\mathrm{CH}_{3}\right)_{5}-2,3,4,5,6\right) ; \quad 58.7 \quad\left(\mathrm{NCH}_{2} \mathrm{CH}_{2} \mathrm{CH}_{2} \mathrm{OCH}_{3}\right) ; \quad 69.0$ $\left(\mathrm{NCH}_{2} \mathrm{CH}_{2} \mathrm{CH}_{2} \mathrm{OCH}_{3}\right) ; 113.1,113.6,125.1,127.0,131.3,131.9,133.6$, 133.9, $137.3\left(\mathrm{NC}_{6} \mathrm{H}_{4} \mathrm{~N}, \mathrm{NCH}_{2} \mathrm{C}_{6}\left(\mathrm{CH}_{3}\right)_{5}-2,3,4,5,6\right) ; 143.2(\mathrm{NCHN})$. IR (cm $\left.{ }^{1}\right) \mathrm{V}_{(\mathrm{CN})}$ : 1556; Anal. Calcd. for $\mathrm{C}_{23} \mathrm{H}_{31} \mathrm{CIN}_{2} \mathrm{O}: \mathrm{C}, 71.39 ; \mathrm{H}, 8.07 ; \mathrm{N}, 7.24$. Found: C, 71.78; H, 8.12; N, 7.29.

1-(3-Methoxypropyl)-3-(4-chlorobenzyl)benzimidazolium chloride (2d): Yield: $1.89 \mathrm{~g}, 90 \%$; mp: $125-126{ }^{\circ} \mathrm{C}$; ${ }^{1} \mathrm{H}$ NMR $\left(500 \mathrm{MHz}, \mathrm{CDCl}_{3}, 25\right.$ $\left.{ }^{\circ} \mathrm{C}\right): \delta 2.35\left(\mathrm{p}, \quad J=5.4 \mathrm{~Hz}, 2 \mathrm{H}, \quad \mathrm{NCH}_{2} \mathrm{CH}_{2} \mathrm{CH}_{2} \mathrm{OCH}_{3}\right) ; 3.26$ (s, $3 \mathrm{H}$, $\mathrm{NCH}_{2} \mathrm{CH}_{2} \mathrm{CH}_{2} \mathrm{OCH}$ ); 3.47 (t, J=5.4 Hz, $2 \mathrm{H}, \mathrm{NCH}_{2} \mathrm{CH}_{2} \mathrm{CH}_{2} \mathrm{OCH}_{3}$ ); 4.71 (t, $\left.J=7.0 \mathrm{~Hz}, 2 \mathrm{H}, \mathrm{NCH}_{2} \mathrm{CH}_{2} \mathrm{CH}_{2} \mathrm{OCH}_{3}\right) ; 5.96\left(\mathrm{~s}, 2 \mathrm{H}, \mathrm{NCH}_{2} \mathrm{C}_{6} \mathrm{H}_{4}(\mathrm{Cl})-4\right) ; 7.29-$ $7.76\left(\mathrm{~m}, 8 \mathrm{H}, \mathrm{NC}_{6} \mathrm{H}_{4} \mathrm{~N}, \mathrm{NCH}_{2} \mathrm{C}_{6} \mathrm{H}_{4}(\mathrm{Cl})-4\right) ; 11.83(\mathrm{~s}, 1 \mathrm{H}, \mathrm{NCHN}) .{ }^{13} \mathrm{C} \mathrm{NMR}$ $\left(125 \mathrm{MHz}, \mathrm{CDCl}_{3}, 25{ }^{\circ} \mathrm{C}\right): \delta \quad 29.6 \quad\left(\mathrm{NCH}_{2} \mathrm{CH}_{2} \mathrm{CH}_{2} \mathrm{OCH}_{3}\right) ; 45.1$ $\left(\mathrm{NCH}_{2} \mathrm{CH}_{2} \mathrm{CH}_{2} \mathrm{OCH}_{3}\right) ; \quad 50.6 \quad\left(\mathrm{NCH}_{2} \mathrm{C}_{6} \mathrm{H}_{4}(\mathrm{Cl})-4\right) ; \quad 58.8$ $\left(\mathrm{NCH}_{2} \mathrm{CH}_{2} \mathrm{CH}_{2} \mathrm{OCH}\right) ; 68.8\left(\mathrm{NCH}_{2} \mathrm{CH}_{2} \mathrm{CH}_{2} \mathrm{OCH}_{3}\right) ; 113.1,113.6,127.1$, 129.5, 129.6, 129.9, 130.9, 131.6, 131.7, $135.2\left(\mathrm{NC}_{6} \mathrm{H}_{4} \mathrm{~N}, \mathrm{NCH}_{2} \mathrm{C}_{6} \mathrm{H}_{4}(\mathrm{Cl})\right.$ 4); $143.8(\mathrm{NCHN})$. IR $\left(\mathrm{cm}^{-1}\right) \mathrm{V}_{(\mathrm{CN})}$ : 1557; Anal. Calcd. for $\mathrm{C}_{18} \mathrm{H}_{20} \mathrm{Cl}_{2} \mathrm{~N}_{2} \mathrm{O}: \mathrm{C}$, $61.55 ; \mathrm{H}, 5.74 ; \mathrm{N}, 7.97$. Found: C, 61.78; H, 5.82; N, 7.99 .

1-(3-Methoxypropyl)-3-(3-methoxylbenzyl)benzimidazolium chloride (2e): Yield: $1.46 \mathrm{~g}, 74 \%$; mp: $95-96{ }^{\circ} \mathrm{C}$; ${ }^{1} \mathrm{H}$ NMR $\left(500 \mathrm{MHz}, \mathrm{CDCl}_{3}, 25\right.$ $\left.{ }^{\circ} \mathrm{C}\right): \delta 2.31\left(\mathrm{p}, J=5.5 \mathrm{~Hz}, 2 \mathrm{H}, \mathrm{NCH}_{2} \mathrm{CH}_{2} \mathrm{CH}_{2} \mathrm{OCH}_{3}\right) ; 3.22(\mathrm{~s}, 3 \mathrm{H}$,
$\mathrm{NCH}_{2} \mathrm{CH}_{2} \mathrm{CH}_{2} \mathrm{OCH}$ ); 3.44 (t, J=5.4 Hz, $2 \mathrm{H}, \mathrm{NCH}_{2} \mathrm{CH}_{2} \mathrm{CH}_{2} \mathrm{OCH}_{3}$ ); 3.75 (s, $3 \mathrm{H}, \mathrm{NCH}_{2} \mathrm{C}_{6} \mathrm{H}_{4}\left(\mathrm{OCH}_{3}\right)-3$ ); 4.72 (t, $J=6.8 \mathrm{~Hz}, 2 \mathrm{H}, \mathrm{NCH}_{2} \mathrm{CH}_{2} \mathrm{CH}_{2} \mathrm{OCH}_{3}$ ); $5.83 \quad\left(\mathrm{~s}, \quad 2 \mathrm{H}, \quad \mathrm{NCH}_{2} \mathrm{C}_{6} \mathrm{H}_{4}\left(\mathrm{OCH}_{3}\right)-3\right) ; \quad 6.79-7.74 \quad\left(\mathrm{~m}, \quad 8 \mathrm{H}, \quad \mathrm{NC}_{6} \mathrm{H}_{4} \mathrm{~N}\right.$, $\left.\mathrm{NCH}_{2} \mathrm{C}_{6} \mathrm{H}_{4}\left(\mathrm{OCH}_{3}\right)-3\right) ; 11.55$ (s, $\left.1 \mathrm{H}, \mathrm{NC} H \mathrm{~N}\right) .{ }^{13} \mathrm{C} \mathrm{NMR}\left(125 \mathrm{MHz}, \mathrm{CDCl}_{3}\right.$, $\left.25{ }^{\circ} \mathrm{C}\right): \delta 29.6\left(\mathrm{NCH}_{2} \mathrm{CH}_{2} \mathrm{CH}_{2} \mathrm{OCH}_{3}\right) ; 45.0\left(\mathrm{NCH}_{2} \mathrm{CH}_{2} \mathrm{CH}_{2} \mathrm{OCH}_{3}\right) ; 51.2$ $\left(\mathrm{NCH}_{2} \mathrm{C}_{6} \mathrm{H}_{4}\left(\mathrm{OCH}_{3}\right)-3\right) ; \quad 55.5 \quad\left(\mathrm{NCH}_{2} \mathrm{C}_{6} \mathrm{H}_{4}\left(\mathrm{OCH}_{3}\right)-3\right) ; \quad 58.7$ $\left(\mathrm{NCH}_{2} \mathrm{CH}_{2} \mathrm{CH}_{2} \mathrm{OCH}\right) ; 68.9\left(\mathrm{NCH}_{2} \mathrm{CH}_{2} \mathrm{CH}_{2} \mathrm{OCH}_{3}\right) ; 113.0,113.7,113.9$, $114.6,120.3,127.0,130.3,131.1,131.7,134.5,160.2\left(\mathrm{NC}_{6} \mathrm{H}_{4} \mathrm{~N}\right.$, $\left.\mathrm{NCH}_{2} \mathrm{C}_{6} \mathrm{H}_{4}\left(\mathrm{OCH}_{3}\right)-3\right) ; 143.7$ (NCHN). IR ( $\left.\mathrm{cm}^{-1}\right) \mathrm{v}_{(\mathrm{CN})}$ : 1558; Anal. Calcd. for $\mathrm{C}_{19} \mathrm{H}_{23} \mathrm{CIN}_{2} \mathrm{O}_{2}$ : C, 65.79; $\mathrm{H}, 6.68 ; \mathrm{N}, 8.08$. Found: $\mathrm{C}, 65.78 ; \mathrm{H}, 6.62 ; \mathrm{N}$, 8.01 .

1-(3-Methoxypropyl)-3-(3,4,5-trimethoxylbenzyl)benzimidazolium chloride (2f): Yield: $2.15 \mathrm{~g}, 91 \%$; mp: $121-122{ }^{\circ} \mathrm{C} ;{ }^{1} \mathrm{H}$ NMR $(500 \mathrm{MHz}$, $\mathrm{CDCl}_{3}, 25^{\circ} \mathrm{C}$ ): $\delta 2.34$ (p, J=5.4 Hz, $2 \mathrm{H}, \mathrm{NCH}_{2} \mathrm{CH}_{2} \mathrm{CH}_{2} \mathrm{OCH}_{3}$ ); 3.24 (s, 3H $\mathrm{NCH}_{2} \mathrm{CH}_{2} \mathrm{CH}_{2} \mathrm{OCH}_{3}$ ); 3.46 (t, $\mathrm{J}=5.3 \mathrm{~Hz}, 2 \mathrm{H}, \mathrm{NCH}_{2} \mathrm{CH}_{2} \mathrm{CH}_{2} \mathrm{OCH}_{3}$ ); 3.79 and $3.85\left(\mathrm{~s}, 9 \mathrm{H}, \mathrm{NCH}_{2} \mathrm{C}_{6} \mathrm{H}_{2}\left(\mathrm{OCH}_{3}\right)_{3}-3,4,5\right) ; 4.72(\mathrm{t}, \mathrm{J}=6.4 \mathrm{~Hz}, 2 \mathrm{H}$, $\mathrm{NCH}_{2} \mathrm{CH}_{2} \mathrm{CH}_{2} \mathrm{OCH}_{3}$ ); 5.80 (s, $\left.2 \mathrm{H}, \mathrm{NCH}_{2} \mathrm{C}_{6} \mathrm{H}_{2}\left(\mathrm{OCH}_{3}\right)_{3}-3,4,5\right) ; 7.57-7.75$ $\left(\mathrm{m}, 6 \mathrm{H}, \mathrm{NC}_{6} \mathrm{H}_{4} \mathrm{~N}, \mathrm{NCH}_{2} \mathrm{C}_{6} \mathrm{H}_{2}\left(\mathrm{OCH}_{3}\right)_{3}-3,4,5\right) ; 11.68(\mathrm{~s}, 1 \mathrm{H}, \mathrm{NCHN}) \cdot{ }^{13} \mathrm{C}$ NMR $\left(125 \mathrm{MHz}, \mathrm{CDCl}_{3}, 2{ }^{\circ} \mathrm{C}\right): \delta 29.6\left(\mathrm{NCH}_{2} \mathrm{CH}_{2} \mathrm{CH}_{2} \mathrm{OCH}_{3}\right) ; 44.9$ $\left(\mathrm{NCH}_{2} \mathrm{CH}_{2} \mathrm{CH}_{2} \mathrm{OCH}_{3}\right) ; 51.7\left(\mathrm{NCH}_{2} \mathrm{C}_{6} \mathrm{H}_{2}\left(\mathrm{OCH}_{3}\right)_{3}-3,4,5\right) ; 56.6$ and 60.8 $\left(\mathrm{NCH}_{2} \mathrm{C}_{6} \mathrm{H}_{2}\left(\mathrm{OCH}_{3}\right)_{3}-3,4,5\right) ; \quad 58.7 \quad\left(\mathrm{NCH}_{2} \mathrm{CH}_{2} \mathrm{CH}_{2} \mathrm{OCH}\right) ; \quad 68.8$ $\left(\mathrm{NCH}_{2} \mathrm{CH}_{2} \mathrm{CH}_{2} \mathrm{OCH}_{3}\right) ; 106.1,113.1,113.6,127.0,128.6,131.1,131.7$, 138.5, $153.8\left(\mathrm{NC}_{6} \mathrm{H}_{4} \mathrm{~N}, \mathrm{NCH}_{2} \mathrm{C}_{6} \mathrm{H}_{2}\left(\mathrm{OCH}_{3}\right)_{3}-3,4,5\right) ; 143.8(\mathrm{NCHN})$. IR (cm $\left.{ }^{1}\right) \mathrm{v}_{(\mathrm{CN})}$ : 1594; Anal. Calcd. for $\mathrm{C}_{21} \mathrm{H}_{27} \mathrm{CIN}_{2} \mathrm{O}_{4}: \mathrm{C}, 61.99 ; \mathrm{H}, 6.69 ; \mathrm{N}, 6.88$. Found: C, 62.03; H, 6.72; N, 6.92

General procedure for the preparation of the PEPPSI-type palladium-NHC complexes: The PEPPSI-type palladium-NHC complexes (3a-f) were obtained in moderate to good yields using the standard procedure initially developed by Organ. ${ }^{[37]} \mathrm{A}$ mixture of $\mathrm{K}_{2} \mathrm{CO}_{3}$ $(5.0 \mathrm{mmol})$, pyridine $(3 \mathrm{~mL}), \mathrm{PdCl}_{2}(1.1 \mathrm{mmol})$, benzimidazolium salt $(1.0$ $\mathrm{mmol})$, and $\mathrm{KBr}(10.0 \mathrm{mmol})$ was heated at $80^{\circ} \mathrm{C}$ for $16 \mathrm{~h}$. The reaction mixture was then filtered through a pad of celite and silica gel to remove the unreacted $\mathrm{PdCl}_{2}$ and benzimidazolium salt. The solvent in the reaction medium was then removed. The resulting complexes were washed with $n$-pentane $(3 \times 5 \mathrm{~mL})$ and dried under vacuum. The crude products were recrystallized from dichloromethane/ $n$-pentane $(1: 2, v / v)$.

trans-Dibromo[1-(3-methoxypropyl)-3-(4-methylbenzyl)benzimidazole-2-ylidene](pyridine)palladium(II) (3a): Yield: $0.12 \mathrm{~g}, 54 \%$; mp: 79-80 ${ }^{\circ} \mathrm{C} ;{ }^{1} \mathrm{H}$ NMR $\left(300 \mathrm{MHz}, \mathrm{CDCl}_{3}, 25^{\circ} \mathrm{C}\right): \delta 2.24\left(\mathrm{~s}, 3 \mathrm{H}, \mathrm{NCH}_{2} \mathrm{C}_{6} \mathrm{H}_{4}\left(\mathrm{CH}_{3}\right)-4\right)$ $2.53\left(\mathrm{p}, \quad J=7.1 \mathrm{~Hz}, \quad 2 \mathrm{H}, \quad \mathrm{NCH}_{2} \mathrm{CH}_{2} \mathrm{CH}_{2} \mathrm{OCH}_{3}\right) ; 3.29 \quad(\mathrm{~s}, \quad 3 \mathrm{H}$, $\mathrm{NCH}_{2} \mathrm{CH}_{2} \mathrm{CH}_{2} \mathrm{OCH}{ }_{3}$ ); 3.44 (t, $\mathrm{J}=5.6 \mathrm{~Hz}, 2 \mathrm{H}, \mathrm{NCH}_{2} \mathrm{CH}_{2} \mathrm{CH}_{2} \mathrm{OCH}_{3}$ ); 4.95 (t $\left.J=7.1 \mathrm{~Hz}, 2 \mathrm{H}, \mathrm{NCH}_{2} \mathrm{CH}_{2} \mathrm{CH}_{2} \mathrm{OCH}_{3}\right) ; 6.10$ (s, $\left.2 \mathrm{H}, \mathrm{NCH}_{2} \mathrm{C}_{6} \mathrm{H}_{4}\left(\mathrm{CH}_{3}\right)-4\right)$; 7.04-7.73 and 8.92-8.95 (m, $13 \mathrm{H}, \quad \mathrm{NC}_{6} \mathrm{H}_{4} \mathrm{~N}, \quad \mathrm{NCH}_{2} \mathrm{C}_{6} \mathrm{H}_{4}\left(\mathrm{CH}_{3}\right)-4$ and $\left.\mathrm{NC}_{5} \mathrm{H}_{5}\right) .{ }^{13} \mathrm{C}$ NMR $\left(75 \mathrm{MHz}, \mathrm{CDCl}_{3}, 25{ }^{\circ} \mathrm{C}\right): \delta 21.2\left(\mathrm{NCH}_{2} \mathrm{C}_{6} \mathrm{H}_{4}\left(\mathrm{CH}_{3}\right)-4\right)$; $30.1 \quad\left(\mathrm{NCH}_{2} \mathrm{CH}_{2} \mathrm{CH}_{2} \mathrm{OCH}_{3}\right) ; \quad 45.1 \quad\left(\mathrm{NCH}_{2} \mathrm{CH}_{2} \mathrm{CH}_{2} \mathrm{OCH}_{3}\right) ; \quad 53.0$ $\left(\mathrm{NCH}_{2} \mathrm{C}_{6} \mathrm{H}_{4}\left(\mathrm{CH}_{3}\right)-4\right) ; \quad 58.6 \quad\left(\mathrm{NCH}_{2} \mathrm{CH}_{2} \mathrm{CH}_{2} \mathrm{OCH}_{3}\right) ; \quad 69.1$ $\left(\mathrm{NCH}_{2} \mathrm{CH}_{2} \mathrm{CH}_{2} \mathrm{OCH}_{3}\right) ; 110.5,111.4,123.1,123.2,124.5,127.9,129.5$ 132.0, 134.0, 135.2, 137.8, 138.2, $151.2\left(\mathrm{NC}_{6} \mathrm{H}_{4} \mathrm{~N}, \mathrm{NCH}_{2} \mathrm{C}_{6} \mathrm{H}_{4}\left(\mathrm{CH}_{3}\right)-4\right.$ and $\left.\mathrm{NC}_{5} \mathrm{H}_{5}\right)$; $163.6\left(\mathrm{Pd}-\mathrm{C}_{\text {carbene }}\right)$. IR $\left(\mathrm{cm}^{-1}\right) \mathrm{V}_{(\mathrm{CN})}$ : 1408; Anal. Calcd. for $\mathrm{C}_{24} \mathrm{H}_{27} \mathrm{Br}_{2} \mathrm{~N}_{3} \mathrm{OPd}$ : C, 45.06; H, 4.25; N, 6.57. Found: C, 45.08; H, 4.27; N 6.59 .

trans-Dibromo[1-(3-methoxypropyl)-3-(4-tert-butylbenzyl)benzimidazole-2-ylidene](pyridine)palladium(II) (3b): Yield: $0.19 \mathrm{~g}, 74 \%$; $\mathrm{mp}$ 109-110 ${ }^{\circ} \mathrm{C} ;{ }^{1} \mathrm{H}$ NMR $\left(300 \mathrm{MHz}, \mathrm{CDCl}_{3}, 25{ }^{\circ} \mathrm{C}\right): \delta 1.21(\mathrm{~s}, 9 \mathrm{H}$, $\left.\mathrm{NCH}_{2} \mathrm{C}_{6} \mathrm{H}_{4}\left(\mathrm{C}\left(\mathrm{CH}_{3}\right)_{3}\right)-4\right) ; 2.54\left(\mathrm{p}, \mathrm{J}=5.6 \mathrm{~Hz}, 2 \mathrm{H}, \mathrm{NCH}_{2} \mathrm{CH}_{2} \mathrm{CH}_{2} \mathrm{OCH}_{3}\right)$; 3.30 (s, $3 \mathrm{H}, \quad \mathrm{NCH}_{2} \mathrm{CH}_{2} \mathrm{CH}_{2} \mathrm{OCH}_{3}$ ); 3.45 (t, $J=5.3 \mathrm{~Hz}, \quad 2 \mathrm{H}$, $\mathrm{NCH}_{2} \mathrm{CH}_{2} \mathrm{CH}_{2} \mathrm{OCH}_{3}$ ); 4.93 (t, $J=7.1 \mathrm{~Hz}, 2 \mathrm{H}, \mathrm{NCH}_{2} \mathrm{CH}_{2} \mathrm{CH}_{2} \mathrm{OCH}_{3}$ ); 6.06 (s, $\left.2 \mathrm{H}, \mathrm{NCH}_{2} \mathrm{C}_{6} \mathrm{H}_{4}\left(\mathrm{C}_{(}\left(\mathrm{CH}_{3}\right)_{3}\right)-4\right)$; 7.00-7.70 and 8.94-8.98 (m, $13 \mathrm{H}, \mathrm{NC}_{6} \mathrm{H}_{4} \mathrm{~N}$, $\mathrm{NCH}_{2} \mathrm{C}_{6} \mathrm{H}_{4}\left(\mathrm{C}_{(}\left(\mathrm{CH}_{3}\right)_{3}\right)-4$ and $\left.\mathrm{NC}_{5} \mathrm{H}_{5}\right) \cdot{ }^{13} \mathrm{C} \mathrm{NMR}\left(75 \mathrm{MHz}, \mathrm{CDCl}_{3}, 25^{\circ} \mathrm{C}\right): \delta$ $28.8 \quad\left(\mathrm{NCH}_{2} \mathrm{CH}_{2} \mathrm{CH}_{2} \mathrm{OCH}_{3}\right) ; \quad 30.3 \quad\left(\mathrm{NCH}_{2} \mathrm{C}_{6} \mathrm{H}_{4}\left(\mathrm{C}_{(}\left(\mathrm{CH}_{3}\right)_{3}\right)-4\right) ; \quad 33.5$ $\left(\mathrm{NCH}_{2} \mathrm{C}_{6} \mathrm{H}_{4}\left(\mathrm{C}\left(\mathrm{CH}_{3}\right)_{3}\right)-4\right) ; \quad 44.3 \quad\left(\mathrm{NCH}_{2} \mathrm{CH}_{2} \mathrm{CH}_{2} \mathrm{OCH}_{3}\right) ; \quad 51.8$ $\left(\mathrm{NCH}_{2} \mathrm{C}_{6} \mathrm{H}_{4}\left(\mathrm{C}\left(\mathrm{CH}_{3}\right)_{3}\right)-4\right) ; \quad 57.6 \quad\left(\mathrm{NCH}_{2} \mathrm{CH}_{2} \mathrm{CH}_{2} \mathrm{OCH}\right)_{3} ; \quad 68.1$ $\left(\mathrm{NCH}_{2} \mathrm{CH}_{2} \mathrm{CH}_{2} \mathrm{OCH}_{3}\right) ; 109.4,110.5,122.0,122.1,123.4,123.5,124.7$, $126.7,126.8,130.9,133.1,137.0,151.0\left(\mathrm{NC}_{6} \mathrm{H}_{4} \mathrm{~N}, \mathrm{NCH}_{2} \mathrm{C}_{6} \mathrm{H}_{4}\left(\mathrm{CH}_{3}\right)-4\right.$ 
and $\left.\mathrm{NC}_{5} \mathrm{H}_{5}\right) ; 162.2\left(\mathrm{Pd}-\mathrm{C}_{\text {carbene }}\right)$. IR $\left(\mathrm{cm}^{-1}\right) \mathrm{v}_{(\mathrm{CN})}$ : 1408; Anal. Calcd. for $\mathrm{C}_{27} \mathrm{H}_{33} \mathrm{Br}_{2} \mathrm{~N}_{3} \mathrm{OPd}$ : C, 47.56; $\mathrm{H}, 4.88 ; \mathrm{N}, 6.16$. Found: $\mathrm{C}, 47.60 ; \mathrm{H}, 4.92 ; \mathrm{N}$, 6.19 .

trans-Dibromo[1-(3-methoxypropyl)-3-(2,3,4,5,6-pentamethylbenzyl) benzimidazole-2-ylidene](pyridine)palladium(II) (3c): Yield: $0.21 \mathrm{~g}$, 63\%; mp: 204-205 ${ }^{\circ} \mathrm{C}$; ${ }^{1} \mathrm{H}$ NMR (300 MHz, $\left.\mathrm{CDCl}_{3}, 25{ }^{\circ} \mathrm{C}\right): \delta 2.25,2.31$ and $2.33\left(\mathrm{~s}, 15 \mathrm{H}, \mathrm{NCH}_{2} \mathrm{C}_{6}\left(\mathrm{CH}_{3}\right)_{5}-2,3,4,5,6\right) ; 2.61(\mathrm{p}, J=6.1 \mathrm{~Hz}, 2 \mathrm{H}$ $\mathrm{NCH}_{2} \mathrm{CH}_{2} \mathrm{CH}_{2} \mathrm{OCH}_{3}$ ); 3.38 (s, 3H, $\mathrm{NCH}_{2} \mathrm{CH}_{2} \mathrm{CH}_{2} \mathrm{OCH}_{3}$ ); 3.52 (t, $J=6.2 \mathrm{~Hz}$ $2 \mathrm{H}, \mathrm{NCH}_{2} \mathrm{CH}_{2} \mathrm{CH}_{2} \mathrm{OCH}_{3}$ ); 5.03 (t, J= $7.1 \mathrm{~Hz}, 2 \mathrm{H}, \mathrm{NCH}_{2} \mathrm{CH}_{2} \mathrm{CH}_{2} \mathrm{OCH}_{3}$ ); $6.27\left(\mathrm{~s}, 2 \mathrm{H}, \mathrm{NCH}_{2} \mathrm{C}_{6}\left(\mathrm{CH}_{3}\right)_{5}-2,3,4,5,6\right) ; 6.40-7.83$ and 8.98-9.00 (m, 9H, $\mathrm{NC}_{6} \mathrm{H}_{4} \mathrm{~N}$ and $\left.\mathrm{NC}_{5} \mathrm{H}_{5}\right) .{ }^{13} \mathrm{C} \mathrm{NMR}\left(75 \mathrm{MHz}, \mathrm{CDCl}_{3}, 25^{\circ} \mathrm{C}\right): \delta 16.9,17.3$ and $17.4 \quad\left(\mathrm{NCH}_{2} \mathrm{C}_{6}\left(\mathrm{CH}_{3}\right)_{5}-2,3,4,5,6\right) ; \quad 30.1 \quad\left(\mathrm{NCH}_{2} \mathrm{CH}_{2} \mathrm{CH}_{2} \mathrm{OCH}_{3}\right) ; \quad 45.2$ $\left(\mathrm{NCH}_{2} \mathrm{CH}_{2} \mathrm{CH}_{2} \mathrm{OCH}_{3}\right) ; \quad 51.2 \quad\left(\mathrm{NCH}_{2} \mathrm{C}_{6}\left(\mathrm{CH}_{3}\right)_{5}-2,3,4,5,6\right) ; \quad 58.6$ $\left(\mathrm{NCH}_{2} \mathrm{CH}_{2} \mathrm{CH}_{2} \mathrm{OCH}_{3}\right) ; 69.2\left(\mathrm{NCH}_{2} \mathrm{CH}_{2} \mathrm{CH}_{2} \mathrm{OCH}_{3}\right) ; 110.2,111.5,122.6$ 123.0, 124.4, 127.8, 133.1, 134.6, 135.0, 135.9, 138.0, $151.2\left(\mathrm{NC}_{6} \mathrm{H}_{4} \mathrm{~N}\right.$, $\mathrm{NCH}_{2} \mathrm{C}_{6}\left(\mathrm{CH}_{3}\right)_{5}-2,3,4,5,6$ and $\left.\mathrm{NC}_{5} \mathrm{H}_{5}\right) ; 163.1\left(\mathrm{Pd}-\mathrm{C}_{\text {carbene }}\right)$. IR $\left(\mathrm{cm}^{-1}\right) \mathrm{v}_{(\mathrm{CN})}$ : 1425; Anal. Calcd. for $\mathrm{C}_{28} \mathrm{H}_{35} \mathrm{Br}_{2} \mathrm{~N}_{3} \mathrm{OPd}$ : C, 48.33; $\mathrm{H}, 5.07 ; \mathrm{N}, 6.04$. Found: C, $48.34 ; \mathrm{H}, 5.07 ; \mathrm{N}, 6.09$.

trans-Dibromo[1-(3-methoxypropyl)-3-(4-chlorobenzyl)benzimidazole-2-ylidene](pyridine)palladium(II) (3d): Yield: $0.10 \mathrm{~g}, 44 \%$; mp: 92-93 ${ }^{\circ} \mathrm{C}$; ${ }^{1} \mathrm{H}$ NMR $\left(300 \mathrm{MHz}, \mathrm{CDCl}_{3}, 25{ }^{\circ} \mathrm{C}\right): \delta 2.52(\mathrm{p}, J=7.0 \mathrm{~Hz}, 2 \mathrm{H}$, $\mathrm{NCH}_{2} \mathrm{CH}_{2} \mathrm{CH}_{2} \mathrm{OCH}_{3}$ ); 3.30 (s, 3H, $\mathrm{NCH}_{2} \mathrm{CH}_{2} \mathrm{CH}_{2} \mathrm{OCH}_{3}$ ); 3.44 (t, $J=5.5 \mathrm{~Hz}$ $2 \mathrm{H}, \mathrm{NCH}_{2} \mathrm{CH}_{2} \mathrm{CH}_{2} \mathrm{OCH}_{3}$ ); 4.93 (t, J= $7.3 \mathrm{~Hz}, 2 \mathrm{H}, \mathrm{NCH}_{2} \mathrm{CH}_{2} \mathrm{CH}_{2} \mathrm{OCH}_{3}$ ); $6.11\left(\mathrm{~s}, 2 \mathrm{H}, \mathrm{NCH}_{2} \mathrm{C}_{6} \mathrm{H}_{4}(\mathrm{Cl})-4\right) ; 7.20-7.75$ and 8.92-8.94 (m, $13 \mathrm{H}, \mathrm{NC}_{6} \mathrm{H}_{4} \mathrm{~N}$ $\mathrm{NCH}_{2} \mathrm{C}_{6} \mathrm{H}_{4}(\mathrm{Cl})-4$ and $\left.\mathrm{NC}_{5} \mathrm{H}_{5}\right) \cdot{ }^{13} \mathrm{C} \mathrm{NMR}\left(75 \mathrm{MHz}, \mathrm{CDCl}_{3}, 2{ }^{\circ} \mathrm{C}\right): \delta 29.0$ $\left(\mathrm{NCH}_{2} \mathrm{CH}_{2} \mathrm{CH}_{2} \mathrm{OCH}_{3}\right) ; 44.2\left(\mathrm{NCH}_{2} \mathrm{CH}_{2} \mathrm{CH}_{2} \mathrm{OCH}_{3}\right) ; 51.4\left(\mathrm{NCH}_{2} \mathrm{C}_{6} \mathrm{H}_{4}(\mathrm{Cl})-\right.$ 4); $57.6\left(\mathrm{NCH}_{2} \mathrm{CH}_{2} \mathrm{CH}_{2} \mathrm{OCH}\right) ; 68.0\left(\mathrm{NCH}_{2} \mathrm{CH}_{2} \mathrm{CH}_{2} \mathrm{OCH}_{3}\right) ; 109.7,110.1$, 122.3, 122.4, 123.6, 128.1, 128.3, 132.5, 132.8, 133.0, 134.1, 137.2, $150.2\left(\mathrm{NC}_{6} \mathrm{H}_{4} \mathrm{~N}, \mathrm{NCH}_{2} \mathrm{C}_{6} \mathrm{H}_{4}(\mathrm{Cl})-4\right.$ and $\left.\mathrm{NC}_{5} \mathrm{H}_{5}\right) ; 163.1$ (Pd- $\left.C_{\text {carbene }}\right)$. IR (cm $\left.{ }^{1}\right) \mathrm{v}_{(\mathrm{CN})}$ : 1405 ; Anal. Calcd. for $\mathrm{C}_{23} \mathrm{H}_{24} \mathrm{Br}_{2} \mathrm{CIN}_{3} \mathrm{OPd}$ : C, 41.85; H, 3.66; N, 6.37. Found: C, $41.87 ; \mathrm{H}, 3.72 ; \mathrm{N}, 6.39$.

\section{trans-Dibromo[1-(3-methoxypropyl)-3-(3-methoxylbenzyl)benzimid-} azole-2-ylidene](pyridine)palladium(II) (3e): Yield: $0.18 \mathrm{~g}, 94 \%$; $\mathrm{mp}$ : 138-139 ${ }^{\circ} \mathrm{C} ;{ }^{1} \mathrm{H}$ NMR $\left(300 \mathrm{MHz}, \mathrm{CDCl}_{3}, 25^{\circ} \mathrm{C}\right): \delta 2.53$ (p, J=6.0 Hz, $2 \mathrm{H}$, $\mathrm{NCH}_{2} \mathrm{CH}_{2} \mathrm{CH}_{2} \mathrm{OCH}_{3}$ ); 3.30 (s, 3H, $\mathrm{NCH}_{2} \mathrm{CH}_{2} \mathrm{CH}_{2} \mathrm{OCH}$ ); 3.45 (t, J=5.6 Hz $2 \mathrm{H}, \mathrm{NCH}_{2} \mathrm{CH}_{2} \mathrm{CH}_{2} \mathrm{OCH}_{3}$ ); 3.66 (s, 3H, $\mathrm{NCH}_{2} \mathrm{C}_{6} \mathrm{H}_{4}\left(\mathrm{OCH}_{3}\right)-3$ ); 4.91 (t, J= $\left.7.2 \mathrm{~Hz}, 2 \mathrm{H}, \mathrm{NCH}_{2} \mathrm{CH}_{2} \mathrm{CH}_{2} \mathrm{OCH}_{3}\right) ; 6.06$ (s, $\left.2 \mathrm{H}, \mathrm{NCH}_{2} \mathrm{C}_{6} \mathrm{H}_{4}\left(\mathrm{OCH}_{3}\right)-3\right) ; 6.06-$ 7.69 and 8.96-8.99 (m, $13 \mathrm{H}, \mathrm{NC}_{6} \mathrm{H}_{4} \mathrm{~N}, \mathrm{NCH}_{2} \mathrm{C}_{6} \mathrm{H}_{4}\left(\mathrm{OCH}_{3}\right)-3$ and $\left.\mathrm{NC}_{5} \mathrm{H}_{5}\right)$ ${ }^{13} \mathrm{C}$ NMR $\left(75 \mathrm{MHz}, \mathrm{CDCl}_{3}, 25{ }^{\circ} \mathrm{C}\right): \delta 29.6\left(\mathrm{NCH}_{2} \mathrm{CH}_{2} \mathrm{CH}_{2} \mathrm{OCH}_{3}\right) ; 45.5$ $\left(\mathrm{NCH}_{2} \mathrm{CH}_{2} \mathrm{CH}_{2} \mathrm{OCH}_{3}\right) ; \quad 53.7 \quad\left(\mathrm{NCH}_{2} \mathrm{C}_{6} \mathrm{H}_{4}\left(\mathrm{OCH}_{3}\right)-3\right) ; \quad 55.7$ $\left(\mathrm{NCH}_{2} \mathrm{C}_{6} \mathrm{H}_{4}\left(\mathrm{OCH}_{3}\right)-3\right) ; \quad 58.6 \quad\left(\mathrm{NCH}_{2} \mathrm{CH}_{2} \mathrm{CH}_{2} \mathrm{OCH}_{3}\right) ; \quad 69.2$ $\left(\mathrm{NCH}_{2} \mathrm{CH}_{2} \mathrm{CH}_{2} \mathrm{OCH}_{3}\right) ; 110.5,111.4,113.0,114.7,120.4,123.0$, 123.1, 123.2, 124.6, 129.7, 134.2, 135.4, 136.5, 137.9, 152.6, $160.2\left(\mathrm{NC}_{6} \mathrm{H}_{4} \mathrm{~N}\right.$ $\mathrm{NCH}_{2} \mathrm{C}_{6} \mathrm{H}_{4}\left(\mathrm{OCH}_{3}\right)-3$ and $\left.\mathrm{NC}_{5} \mathrm{H}_{5}\right) ; 163.1$ (Pd- $C_{\text {carbene) }}$. IR ( $\left.\mathrm{cm}^{-1}\right) \mathrm{V}_{(\mathrm{CN})}$ : 1412; Anal. Calcd. for $\mathrm{C}_{24} \mathrm{H}_{27} \mathrm{Br}_{2} \mathrm{~N}_{3} \mathrm{O}_{2} \mathrm{Pd}$ : C, 43.96; $\mathrm{H}, 4.15$; N, 6.41 . Found: C, 43.95; H, 4.15; N, 6.40 .

trans-Dibromo[1-(3-methoxypropyl)-3-(3,4,5-trimethoxylbenzyl)benzimidazole-2-ylidene](pyridine)palladium(II) (3f): Yield: $0.26 \mathrm{~g}, 78 \%$; mp: $176-177^{\circ} \mathrm{C} ;{ }^{1} \mathrm{H}$ NMR $\left(300 \mathrm{MHz}, \mathrm{CDCl}_{3}, 25^{\circ} \mathrm{C}\right): \delta 2.62$ (p, J=7.2 Hz, $2 \mathrm{H}, \mathrm{NCH}_{2} \mathrm{CH}_{2} \mathrm{CH}_{2} \mathrm{OCH}_{3}$ ); 3.39 (s, 3H, $\mathrm{NCH}_{2} \mathrm{CH}_{2} \mathrm{CH}_{2} \mathrm{OCH}_{3}$ ); 3.53 (t, J= $\left.5.6 \mathrm{~Hz}, 2 \mathrm{H}, \mathrm{NCH}_{2} \mathrm{CH}_{2} \mathrm{CH}_{2} \mathrm{OCH}_{3}\right) ; 3.84\left(\mathrm{~s}, 9 \mathrm{H}, \mathrm{NCH}_{2} \mathrm{C}_{6} \mathrm{H}_{2}\left(\mathrm{OCH}_{3}\right)_{3}-3,4,5\right)$; 5.00 (t, $\left.J=7.2 \mathrm{~Hz}, 2 \mathrm{H}, \quad \mathrm{NCH}_{2} \mathrm{CH}_{2} \mathrm{CH}_{2} \mathrm{OCH}_{3}\right) ; 6.10 \quad(\mathrm{~s}, \quad 2 \mathrm{H}$, $\left.\mathrm{NCH}_{2} \mathrm{C}_{6} \mathrm{H}_{2}\left(\mathrm{OCH}_{3}\right)_{3}-3,4,5\right)$; 6.87-7.83 and 9.06-9.08 (m, $11 \mathrm{H}, \mathrm{NC}_{6} \mathrm{H}_{4} \mathrm{~N}$, $\mathrm{NCH}_{2} \mathrm{C}_{6} \mathrm{H}_{2}\left(\mathrm{OCH}_{3}\right)_{3}-3,4,5$ and $\left.\mathrm{NC}_{5} \mathrm{H}_{5}\right) .{ }^{13} \mathrm{C} \mathrm{NMR}\left(75 \mathrm{MHz}, \mathrm{CDCl}_{3}, 25{ }^{\circ} \mathrm{C}\right)$ : $\delta \quad 29.6\left(\mathrm{NCH}_{2} \mathrm{CH}_{2} \mathrm{CH}_{2} \mathrm{OCH}_{3}\right) ; \quad 45.5 \quad\left(\mathrm{NCH}_{2} \mathrm{CH}_{2} \mathrm{CH}_{2} \mathrm{OCH}_{3}\right) ; \quad 54.0$ $\left(\mathrm{NCH}_{2} \mathrm{C}_{6} \mathrm{H}_{2}\left(\mathrm{OCH}_{3}\right)_{3}-3,4,5\right) ; 56.6$ and $60.8\left(\mathrm{NCH}_{2} \mathrm{C}_{6} \mathrm{H}_{2}\left(\mathrm{OCH}_{3}\right)_{3}-3,4,5\right)$; $58.6\left(\mathrm{NCH}_{2} \mathrm{CH}_{2} \mathrm{CH}_{2} \mathrm{OCH}\right) ; 69.1\left(\mathrm{NCH}_{2} \mathrm{CH}_{2} \mathrm{CH}_{2} \mathrm{OCH}_{3}\right) ; 105.3,110.5$, 111.4, 123.1, 123.2, 124.6, 130.6, 134.2, 135.4, 137.7, 138.0, 152.6, $153.6\left(\mathrm{NC}_{6} \mathrm{H}_{4} \mathrm{~N}, \mathrm{NCH}_{2} \mathrm{C}_{6} \mathrm{H}_{2}\left(\mathrm{OCH}_{3}\right)_{3}-3,4,5\right.$ and $\left.\mathrm{NC}_{5} \mathrm{H}_{5}\right) ; 163.0$ (Pd- $C_{\text {carbene) }}$. IR $\left(\mathrm{cm}^{-1}\right) \mathrm{v}_{(\mathrm{CN})}$ : 1410; Anal. Calcd. for $\mathrm{C}_{26} \mathrm{H}_{31} \mathrm{Br}_{2} \mathrm{~N}_{3} \mathrm{O}_{4} \mathrm{Pd}$ : C, 43.63; $\mathrm{H}$, 4.37; N, 5.87. Found: C, 43.68; H, 4.42; N, 5.91 .

General procedure for the arylation reaction: An oven dried Schlenk flask was charged with KOAc $(1.0 \mathrm{mmol})$, aryl halide $(1.0 \mathrm{mmol}), 2$ - substituted thiophene derivative $(2.0 \mathrm{mmol})$, Pd-NHC-PEPPSI complexes 3a-f $(1.0 \mathrm{~mol} \%)$. Then degassed DMAc $(2 \mathrm{~mL})$ was added under an argon stream. The Schlenk flask was placed in a preheated oil bath at $150^{\circ} \mathrm{C}$, and the reaction mixture was stirred for different durations given in the specific tables. At the end of the reaction, the solvent was removed under vacuum, and the residue was charged directly onto a silica gel column. The products were eluted from $n$-hexane/diethylether mixture $(5: 1, v / v)$. The chemical characterizations of the products were made by GC and GC-MS. The convertions were based on aryl halides.

X-ray Crystallographic Data: Single crystals of complex $3 \mathbf{f}$ suitable for $X$-ray analysis were obtained by slow diffusion of $n$-pentane into a dichloromethane solution of the complex 3f. Empirical formula= $\mathrm{C}_{26} \mathrm{H}_{31} \mathrm{Br}_{2} \mathrm{~N}_{3} \mathrm{O}_{4} \mathrm{Pd}$; $M=715.76$ g.mol ${ }^{-1}$; crystal color= yellow; crystal system $=$ monoclinic; space group $=P 12_{1} / c 1$ system; $a=7.4450(3), b=$ 45.231(2), $c=8.8912(5) \AA, \beta=111.745(2)^{\circ}, V=2781.0(2) \AA^{3} . Z=4, d=$ $1.710 \mathrm{~g} \cdot \mathrm{cm}^{-3}, \mu=3.577 \mathrm{~mm}^{-1} . F(000)=1424 ; T=150(2) \mathrm{K}$. The crystal size $(0.340 \times 0.200 \times 0.150 \mathrm{~mm})$ was studied with an D8 VENTURE Bruker AXS diffractometer. Mo-Ka radiation $(\lambda=0.71073 \AA)$. The structure was solved by direct methods using the SIR97 program ${ }^{[40]}$ and then refined with full-matrix least-square methods based on $F^{2}$ (SHELXL97). ${ }^{[41]}$ All non-hydrogen atoms were refined with anisotropic atomic displacement parameters. $\mathrm{H}$ atoms were finally included in their calculated positions. A final refinement on $F^{2}$ with 6350 unique intensities and 329 parameters converged at $\omega R\left(F^{2}\right)=0.1438(R(F)=0.0645)$ for 5558 observed reflections with $I>2 \sigma(I) . \quad W=1 /\left[\sigma\left(F_{0}^{2}\right)+a P^{2}+b P\right]$ where $P=\left[2 F_{\mathrm{c}}^{2}+\operatorname{MAX}\left(F_{0}^{2}, 0\right)\right] / 3$.

CCDC reference number is 1510296 (for complex 3f) contain the supplementary crystallographic data for this paper. This data can be obtained free of charge from The Cambridge Crystallographic Data Centre.

Supporting Information (see footnote on the first page of this article): for the ${ }^{1} \mathrm{H}$ NMR, ${ }^{13} \mathrm{C}$ NMR and IR spectra of benzimidazolium salts (2a-f) and PEPPSI-type palladium-NHC complexes (3a-f).

\section{Acknowledgements}

This work was financially supported by the Technological Scientific Research Council of Turkey TÜBITAK-BOSPHORUS (France) [109T605] and İnönü University Research Fund (İ .Ü. B.A.P: 2015/41-Güdümlü).

Keywords: $N$-heterocyclic carbenes - benzimidazolium salts PEPPSI-type palladium-NHC complexes - heteroarenes • substituted thiophenes $\cdot$ aryl halides $\bullet$ direct arylation

[1] K. Öfele, J. Organomet. Chem.1968, 12, 42-43.

[2] H.-W. Wanzlick, H.-J. Schönherr, Angew. Chem. Int. Ed. Engl. 1968, 7, 141-142.

[3] a) D. J. Cardin, B. Çetinkaya, M. F. Lappert, Chem. Rev. 1972, 72, 545 574 ; b) B. Çetinkaya, P. H. Dixneuf, M. F. Lappert, J. Chem. Soc Chem. Commun. 1973, 206-207; c) B. Çetinkaya, P. H. Dixneuf, M. F Lappert, J. Chem. Soc. Dalton Trans. 1974, 1827-1833.

[4] A. J. Arduengo III, R. L. Harlow, M. Kline, J. Am. Chem. Soc. 1991, 113, 361-363.

[5] W. A. Herrmann, Angew. Chem. Int. Ed. 2002, 41, 1290-1309.

[6] S. Demir, İ. Özdemir, B. Çetinkaya, H. Arslan, D. VanDerveer, Polyhedron, 2011, 30, 195-200.

[7] E. Peris, R. H. Crabtree, Coord. Chem. Rev. 2004, 248, 2239-2246.

[8] N. Gürbüz, E. Ö. Karaca, İ. Özdemir, B. Çetinkaya, Turk J. Chem. 2015, 39, 1115-1157.

[9] N. Hadei, E. A. B. Kantchev, C. J. O'Brien, M. G. Organ, Org. Lett. 2005, 7, 1991-1994. 
[10] V. Paradiso, C. Costabile, F. Grisi, Molecules, 2016, 21, 117.

[11] Y. Borguet, G. Zaragoza, A. Demonceaua, L. Delaude, Dalton Trans. 2013, 42, 7287-7296.

[12] F. Rajabi, W. R. Thiel, Adv. Synth. Catal. 2014, 356, 1873-1877.

[13] M. G. Organ, M. Abdel-Hadi, S. Avola, I. Dubovyk, N. Hadei, E. A. B. Kantchev, C. J. O'Brien, M. Sayah, C. Valente, Chem. Eur. J. 2008, 14 2443-2452.

[14] S. Burling, M. K. Whittlesey and J. M. J. Williams, Adv. Synth. Catal. 2005 347, 591-594.

[15] G. C. Fortman, S. P. Nolan, Chem. Soc. Rev. 2011, 40, 5151-5169.

[16] F. E. Hahn; Angew. Chem. Int. Ed. 2006, 45, 1348-1352.

[17] D. Bourissou, O. Guerret, F.P. Gabbai, G. Bertrand, Chem. Rev. 2000 , 100, 39-92.

[18] a) Y. Akita, A. Inoue, K. Yamamoto, A. Ohta, T. Kurihara, M. Shimizu, Heterocycles, 1985, 23, 2327-2333; b) A. Ohta, Y. Akita, T. Ohkawa, M. Shiba, R. Fukunaga, A. Miyafuji, T. Nakata, N. Tani, Y. Aoyagi, Heterocycles, 1990, 31, 1951-1958.

[19] C.-L. Sun, B.-J. Li, Z.-J. Shi, Chem. Commun. 2010, 46, 677-685

[20] P. Xi, F. Yang, S. Qin, D. Zhao, J. Lan, G. Gao, C. Hu, J. You, J. Am Chem. Soc. 2010, 132, 1822-1824.

[21] N. Lebrasseur, I. Larrosa, J. Am. Chem. Soc. 2008, 130, 2926-2927.

[22] M. Ionita, J. Roger, H. Doucet, ChemSusChem, 2010, 3, 367-376.

[23] Z. Jin, S.-X. Guo, L.-L. Qiu, G.-P. Wu, J.-X. Fang, Appl. Organomet. Chem. 2011, 25, 502-507.

[24] T. He, M. Wang, P. Li, L. Wang, Chin. J. Chem. 2012, 30, 979-984.

[25] R. Rossi, M. Lessi, C. Manzini, G. Marianetti, Adv. Synth. Catal. 2015, 357, 3777-3814.

[26] A. J. Cresswell, G. C. Lloyd-Jones, Chem. Eur. J. 2016, 22, 12641-12645.

[27] S. Vásquez-Céspedes, K. M. Chepiga, N. Möller, A. H. Schäfer, F. Glorius ACS Catal. 2016, 6, 5954-5961.

[28] T. Patra, S. Nandi, S. K. Sahoo, D. Maiti, Chem. Commun. 2016, 52, 1432-1435.

[29] a) İ. Özdemir, N. Gürbüz, N. Kaloğlu, Ö. Doğan, M. Kaloğlu, C. Bruneau, H. Doucet, Beilstein J. Org. Chem. 2013, 9, 303-312; b) İ. Özdemir, S. Demir, B. Çetinkaya, C. Gourlaouen, F. Maseras, C. Bruneau, P. H Dixneuf, J. Am. Chem. Soc. 2008, 130, 1156-1157; c) N. Gürbüz, I. Özdemir, B. Çetinkaya, Tetrahedron Lett. 2005, 46, 2273-2277; d) M Yiğit, İ. Özdemir, E. Çetinkaya, B. Çetinkaya, Transition Met. Chem. 2007, 32, 536-540; e) İ. Özdemir, S. Demir, B. Çetinkaya, E. Çetinkaya J. Organomet. Chem. 2005, 690, 5849-5855; f) I. Özdemir, Y. Gök, Ö Özeroğlu, M. Kaloğlu, H. Doucet, C. Bruneau, Eur. J. Inorg. Chem. 2010, 1798-1805; (g) E. Ö. Karaca, N. Gürbüz, İ. Özdemir, H. Doucet O. Şahin, O. Büyükgüngör, B. Çetinkaya, Organometallics, 2015, 34 2487-2493.

[30] a) M. Thevenin, S. Thoret, P. Grellier, J. Dubois, Bioorg. Med. Chem. 2012, 21, 4885-4892; b) C. Salome, N. Ribeiro, T. Chavagnan, F. Thuaud, M. Serova, A. de Gramont, S. Faivre, E. Raymond, L. Desaubry, Eur. J. Med. Chem. 2014, 81, 181-191; c) Y. Xia, Y. L. Jin, N Kaur, Y. Choi, K. Lee, Eur. J. Med. Chem. 2011, 46, 2386-2396; d) F. O'Hara, A. C. Burns, M. R. Collins, D. Dalvie, M. A. Ornelas, A. D. N Vaz, Y. Fujiwara, P. S. Baran, J. Med. Chem. 2014, 57, 1616-1620; e) J. P. Michael, Nat. Prod. Rep. 2005, 22, 627-646; f) M. Schlosser, F. Mongin, Chem. Soc. Rev. 2007, 36, 1161-1172; g) D. Zhao, J. You and C. Hu, Chem. Eur. J. 2011, 17, 5466-5492; h) J. S. Carey, D. Laffan, C. Thomson, M. T. Williams, Org. Biomol. Chem. 2006, 4, 2337-2347; i) Y. Liu, S. Zhang, P. J. M. Abreu, Nat. Prod. Rep. 2006, 23, 630-651; j) F. Derridj, J. Roger, S. Djebbar, H. Doucet, Org. Lett. 2010, 12(19), 43204323; k) J. J. Dong, H. Doucet, Eur. J. Org. Chem. 2010, 611-615.
[31] a) E. C. Chao, Drugs Future, 2011, 36, 351-357; b) C. D. Jones, M. G. Jevnikar, A. J. Pike, M. K. Peters, L. J. Black, A. R. Thompson, J. F Falcone, J. A. Clemens, J. Med. Chem. 1984, 27, 1057-1066; c) A. W. Herling, T. Scholl, M. Bickel, H. J. Lang, K. H. Scheunemann, K. Weidmann, R. Rippel; Pharmacology, 1991, 43, 293-303; d) J. Maj, Z. Rogoz, H. Sowinska, Z. Zalewski, J. Pol; Pharmacol. Pharm. 1987, 39 63-74; e) V. Schulz, W. Fischer, U. Hanselle, W. Huhmann, V. Zietsch, Eur. J. Clin. Pharmacol. 1986, 31, 411-414.

[32] a) S. Yanagisawa, K. Ueda, H. Sekizawa, K. Itami, J. Am. Chem. Soc 2009, 131, 14622-14623; b) K. Ueda, S. Yanagisawa, J. Yamaguchi, K. Itami, Angew. Chem. 2010, 49, 8946-8949; c) J. Roger, A. L. Gottumukkala, H. Doucet, ChemCatChem, 2010, 2, 20-40.

[33] N. M. Scott, S. P. Nolan in N-Heterocyclic Carbenes in Synthesis (Eds.: S. P. Nolan), Wiley-VCH, Weinheim, 2006, pp. 55-70.

[34] a) O. Diebolt, P. Braunstein, S. P. Nolan, C. S. J. Cazin, Chem. Commun 2008, 3190-3192; b) A. Chartoire, M. Lesieur, L. Falivene, A. M. Z Slawin, L. Cavallo, C. S. J. Cazin, S. P. Nolan, Chem. Eur. J. 2012, 18 4517-4521; c) I. Maluenda, O. Navarro, Molecules 2015, 20(5), 7528 7557; d) D. Guest, V. H. Menezes da Silva, A. P. de Lima Batista, S. M. Roe, A. A. C. Braga,O. Navarro, Organometallics 2015, 34, 2463-2470 e) P. G. Gildner, T. J. Colacot, Organometallics 2015, 34, 5497-5508; f) A. J. DeAngelis, P. G. Gildner, R. Chow, T. J. Colacot, J. Org. Chem. 2015, 80, 6794-6813.

[35] a) C. Valente, M. Pompeo, M. Sayah, M. G. Organ, Org. Process Res. Dev. 2014, 18, 180-190; b) X.-X. He, Y. Li, B.-B. Ma, Z. Ke, F.-S. Liu, Organometallics, 2016, 35, 2655-2663; c) M. Kaloğlu, D. Sémeril, E Brenner, D. Matt, I. Özdemir, L. Toupet, Eur. J. Inorg. Chem. 2016, 1115-1120; d) S. Yaşar, Ç. Şahin, M. Arslan, İ. Özdemir, J. Organomet. Chem. 2015, 776, 107-112.

[36] C. J. O'Brien, E. A. B. Kantchev, C. Valente, N. Hadei, G. A. Chass, A Lough, A. C. Hopkinson, M. G. Organ, Chem. Eur. J. 2006, 12, 4743 4748

[37] a) M. Sayah, A. J. Lough, M. G. Organ, Chem. Eur. J. 2013, 19, 2749 2756; b) A. Chartoire, X. Frogneux, A. Boreux, A. M. Z. Slawin, S. P. Nolan, Organometallics, 2012, 31, 6947-6951; c) M. G. Organ, S Çalimsiz, M. Sayah, K. H. Hoi, A. J. Lough, Angew. Chem. 2009, 121, 2419-2423; d) J. Zhao, L. Yang, K. Ge, Q. Chen, Y. Zhuang, C. Cao, Y Shi, Inorg. Chem. Commun. 2012, 20, 326-329.

[38] a) K. -T. Chan, Y. -H. Tsai, W. -S. Lin, J. -R. Wu, S. -J. Chen, F. -X. Liao, C. -H. Hu, H. M. Lee, Organometallics, 2010, 29, 463-472; b) X. -X He, Y. Li, B. -B. Ma, Z. Ke, F. -S. Liu, Organometallics, 2016, 35, 2655 2663; c) S. Otsuka, H. Yorimitsu, A. Osuka, Chem. Eur. J. 2015, 21, 14703-14707; d) M. N. Hopkinson, C. Richter, M. Schedler, F. Glorius Nature, 2014, 510, 485-496.

[39] a) M. Kaloğlu, N. Kaloğlu, İ. Özdemir, S. Günal, İ. Özdemir, Bioorg. Med Chem. 2016, 24, 3649-3656; b) S. Günal N. Kaloğlu, İ. Özdemir, S. Demir, İ. Özdemir, Inorg. Chem. Commun. 2012, 21, 142-146; c) Y Gök, N. Gürbüz, İ. Özdemir, B. Çetinkaya, E. Çetinkaya, Appl. Organometal. Chem. 2005, 19, 870-874; d) A. K. Lal, M. D. Milton Sensors and Actuators B, 2014, 202, 257-262; e) İ. Ozdemir, S. Demir Düşünceli, N. Kaloğlu, M. Achard, C. Bruneau, J. Organomet. Chem. 2015, 799-800, 311-315.

[40] A. Altomare, M. C. Burla, M. Camalli, G. Cascarano, C. Giacovazzo, A Guagliardi, A. G. G. Moliterni, G. Polidori, R. Spagna, J. Appl. Cryst. 1999, 32, 115-119.

[41] G. M. Sheldrick, Acta Cryst. A64, 2008, 112-122. 


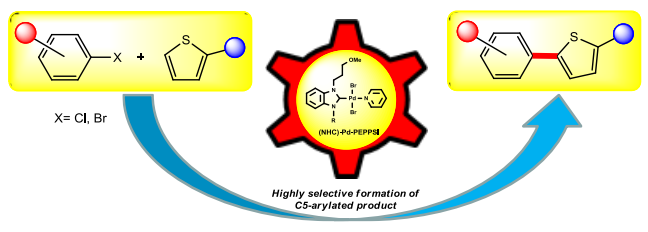

PEPPSI-type palladium-NHC complexes have been synthesized and the catalytic activity of the complexes have been evaluated in the direct C5-arylation of 2substituted thiophene derivatives with various aryl bromides and aryl chlorides
Palladium-NHC-PEPPSI Complexes: Efficient Catalsts For The Direct C5Arylation of 2-Substituted Thiophenes

M. Kaloğlu, I. Özdemir* V. Dorcet, C. Bruneau, H. Doucet

$1-10$

Palladium-NHC-PEPPSI Complexes: Synthesis, Characterization and Catalytic Activity in the Direct C5Arylation of 2-Substituted Thiophene 